\title{
Vertical temperature profile and mesospheric winds retrieval on Mars from CO millimeter observations Comparison with general circulation model predictions
}

\author{
T. Cavalié ${ }^{1,2}$, F. Billebaud ${ }^{1,2}$, T. Encrenaz ${ }^{3}$, M. Dobrijevic ${ }^{1,2}$, J. Brillet ${ }^{1,2}$, F. Forget $^{4}$, and E. Lellouch $^{3}$ \\ 1 Université Bordeaux I, Laboratoire d'Astrophysique de Bordeaux, France \\ e-mail: cavalie@obs.u-bordeaux1.fr \\ 2 CNRS/INSU, UMR5804, BP 89, 33270 Floirac, France \\ 3 Observatoire de Paris-Meudon, Laboratoire d'Études Spatiales et d'Instrumentation en Astrophysique, France \\ ${ }^{4}$ Université Paris VI, Institut Pierre Simon Laplace, Laboratoire de Météorologie Dynamique, France
}

Received 20 March 2008 / Accepted 3 June 2008

\section{ABSTRACT}

\begin{abstract}
Aims. We have recorded high spectral resolution spectra and derived precise atmospheric temperature profiles and wind velocities in the atmosphere of Mars. We have compared observations of the planetary mean thermal profile and mesospheric wind velocities on the disk, obtained with our millimetric observations of CO rotational lines, to predictions from the Laboratoire de Météorologie Dynamique (LMD) Mars General Circulation Model, as provided through the Mars Climate Database (MCD) numerical tool.

Methods. We observed the atmosphere of Mars at $\mathrm{CO}(1-0)$ and $\mathrm{CO}(2-1)$ wavelengths with the IRAM 30-m antenna in June 2001 and November 2005. We retrieved the mean thermal profile of the planet from high and low spectral resolution data with an inversion method detailed here. High spectral resolution spectra were used to derive mesospheric wind velocities on the planetary disk. We also report here the use of ${ }^{13} \mathrm{CO}(2-1)$ line core shifts to measure wind velocities at $40 \mathrm{~km}$.

Results. Neither the Mars Year 24 (MY24) nor the Dust Storm scenario from the Mars Climate Database (MCD) provides satisfactory fits to the 2001 and 2005 data when retrieving the thermal profiles. The Warm scenario only provides good fits for altitudes lower than $30 \mathrm{~km}$. The atmosphere is warmer than predicted up to $60 \mathrm{~km}$ and then becomes colder. Dust loading could be the reason for this mismatch. The MCD MY24 scenario predicts a thermal inversion layer between 40 and $60 \mathrm{~km}$, which is not retrieved from the high spectral resolution data. Our results are generally in agreement with other observations from 10 to $40 \mathrm{~km}$ in altitude, but our results obtained from the high spectral resolution spectra differ in the 40-70 km layer, where the instruments are the most sensitive. The wind velocities we retrieve from our ${ }^{12} \mathrm{CO}$ observations confirm MCD predictions for 2001 and 2005. Velocities obtained from ${ }^{13} \mathrm{CO}$ observations are consistent with MCD predictions in 2001, but are lower than predicted in 2005.
\end{abstract}

Key words. planets and satellites: individual: Mars - radio lines: solar system

\section{Introduction}

The atmosphere of Mars is mainly composed of carbon dioxide. Its molar fraction is about 0.953 (Owen et al. 1977). $\mathrm{CO}_{2}$ photolysis is the source of carbon monoxide. Since its first detection (Kaplan et al. 1969), its abundance vertical profile has been studied in order to understand the recycling of $\mathrm{CO}$ into $\mathrm{CO}_{2}$. Observations have been carried out either in the millimeter (Clancy et al. 1983; Lellouch et al. 1991b; Encrenaz et al. 2001), in the submillimeter (Lellouch et al. 1991a; Gurwell et al. 2000) or in the infrared range (Billebaud et al. 1992, 1998). These observations led to the conclusion that $\mathrm{CO}$ has a relatively stable mixing ratio $q_{\mathrm{CO}}=8 \pm 2 \times 10^{-4}$, both spatially and temporally. However, variations of less than $40 \%$ in magnitude were suggested over the Martian disk (Lellouch et al. 1991b). Then, Krasnopolsky (2003) characterized a north/south asymmetry of the $\mathrm{CO}$ mixing ratio for $L_{\mathrm{S}}=112^{\circ}$. Variations of $q_{\mathrm{CO}}$ going from $8.3 \times 10^{-4}$ northward of the subsolar latitude up $12.5 \times 10^{-4}$ at $50^{\circ} \mathrm{S}$ were observed. Krasnopolsky (1993) showed that there are also variations of the disk-averaged value of $q_{\mathrm{CO}}$ related to the solar cycle $\left(5.8 \times 10^{-4}\right.$ two years after the solar minimum and $8.2 \times 10^{-4}$ two years after the solar maximum). All the previously cited measurements were carried out with a low spatial resolution, except Krasnopolsky (2003). A recent study of Omega/Mars Express data showed that variations as a function of the seasonal cycle of the $\mathrm{CO}$ mixing ratio could reach a factor of 2 above the Hellas Basin (Encrenaz et al. 2006). It is also likely that the atmosphere above the southern condensing polar cap is enriched in $\mathrm{CO}$, similarly to other non-condensable gas such as argon for which this enrichment (by a factor of at least 6) has been detected by Mars Odyssey (Sprague et al. 2004).

In addition to the determination of the $\mathrm{CO}$ mixing ratio, CO observations can also be used to constrain the thermal profile of the planet (Lellouch et al. 1989; Billebaud et al. 1992). In the millimeter wavelength range, the ${ }^{12} \mathrm{CO}$ lines are optically thick and thus the spectral shapes are governed by the atmospheric temperature. The thermal profile can be obtained from the inversion of the spectra. Such observations need to be combined with isotopic observations (optically thin lines) in order to retrieve the $\mathrm{CO}$ mixing ratio at the same time. This is the reason why ${ }^{12} \mathrm{CO}$ and ${ }^{13} \mathrm{CO}$ lines are usually observed jointly. Subsequent analysis of other species generally require knowledge of the thermal profile in order to constrain the vertical profiles of these species (Gurwell et al. 2000; Encrenaz et al. 2001). Any uncertainty in the shape of the temperature profile induces 
uncertainties in the mixing ratio profiles. Therefore, deriving a precise thermal profile is of key importance in any mixing ratio vertical profile study.

Measuring wind velocities in the atmosphere of Mars can be achieved from infrared heterodyne observations of $\mathrm{CO}_{2}$ (Sonnabend et al. 2006). Also, observing line cores at millimeter and submillimeter wavelengths with high spectral and spatial resolution is an effective tool to measure atmospheric wind velocities. Such observations were performed with radio interferometers (Shah et al. 1991; Moreno et al. 2006), but can also be done from single dish antennas (Lellouch et al. 1991c), when the size of the planet is great enough with respect to the beam size. Such conditions are fulfilled at opposition. The measured winds are obtained in regions where the line cores are formed. In the case of $\mathrm{CO}$, the line cores are formed between 40 and $80 \mathrm{~km}$, depending on the isotope and frequency of the line that is observed (Lellouch et al. 1991c; Clancy et al. 2006). Such observations are all the more valuable as this region of the Martian atmosphere is poorly constrained by general circulation models.

The 2001 and 2005 oppositions were good opportunities for Mars observations because the apparent size of the planet resulted in a good spatial resolution when using the Institut de Radio-Astronomie Millimétrique 30-m antenna. The planet was observed a few days before the global dust storm in 2001 and a few days after a regional dust storm in 2005 . We used ${ }^{12} \mathrm{CO}$ and ${ }^{13} \mathrm{CO}$ millimetric line observations to derive the planetary mean atmospheric thermal profile with a combination of high and low spectral resolution spectra. A 13 point map was constructed and wind maps derived from the high spectral resolution ${ }^{12} \mathrm{CO}$ and ${ }^{13} \mathrm{CO}(2-1)$ observations. We present here the wind measurements from ${ }^{12} \mathrm{CO}(2-1)$ and ${ }^{13} \mathrm{CO}(2-1)$ spectra. A description of the observations is given in Sect. 2. The thermal profile retrieval for the central position of our observations is described in Sect. 3. The profiles are compared to other observations and to the predictions from the LMD General Circulation Model (Forget et al. 1999) available using the Mars Climate Database (MCD) numerical tool (Lewis et al. 1999; Forget et al. 2006). The wind measurements are presented in Sect. 4 and also compared to MCD predictions. Our conclusions are given in Sect. 5.

\section{Observations}

The data presented in this paper were obtained during the periods of the 2001 and 2005 oppositions, on 13-17 June 2001 and 31 October 2005 at the Institut de Radio-Astronomie Millimétrique 30-m antenna at Pico Veleta, Spain. The solar longitude $\left(L_{\mathrm{S}}\right)$ values, the planet apparent size and the planetocentric coordinates of the sub-earth point (SEP) at the time of the observations are displayed in Table 1. The antenna was pointed towards 13 points on the planetary disk (see Tables 2, 3 and Fig. 1). We chose the location of the 13 points in order to have a good sampling of the Martian disk with the antenna beam at 230-GHz. Each pointing position was observed $80 \mathrm{~s}$ and then cycled through the whole observing run.

There are two causes of smearing in the data. The first one is the antenna beam width (half of the size of the planet). The other one is the change of the SEP position during an observing run and from one observing run to another. The dates given in Table 1 are the median dates for both sets of observations. We chose to perform our computations at these dates because the general thermal structure of the atmosphere and surface does not vary much for a fixed local time (midday for example) on the visible disk. This is confirmed by the fact that all the datasets gave very similar spectra (contrast, width ...), at each point on
Table 1. The solar longitude $\left(L_{\mathrm{S}}\right)$ values, the planet apparent size and the planetocentric coordinates of the sub-earth point (SEP) at the time of the observations.

\begin{tabular}{ccc}
\hline \hline$L_{\mathrm{S}}$ & 15 June 2001 & 1 November 2005 \\
& $180^{\circ}$ & $316^{\circ}$ \\
angular size & (northern autumn equinox) & (northern winter) \\
SEP at 0h UTC & 20.6 arcsec & 20.2 arcsec \\
\hline
\end{tabular}

Table 2. Coordinates of and local time at the observed points on the planetary disk in 2001. Celestial offsets.

\begin{tabular}{cccc}
\hline \hline Position number & \multicolumn{2}{c}{ Offsets ["] } & Approximate local time \\
& East-West & North-South \\
\hline 1 & 0.0 & 0.0 & $12 \mathrm{~h}$ \\
2 & 2.7 & 4.7 & $12 \mathrm{~h}$ \\
3 & 5.1 & 8.9 & $12 \mathrm{~h}$ \\
4 & -4.7 & 2.2 & $14 \mathrm{~h}$ \\
5 & -8.9 & 5.2 & $18 \mathrm{~h}$ \\
6 & -2.8 & -4.9 & $12 \mathrm{~h}$ \\
7 & -5.1 & -8.8 & $12 \mathrm{~h}$ \\
8 & 4.2 & -3.0 & $10 \mathrm{~h}$ \\
9 & 8.9 & -5.2 & $6 \mathrm{~h}$ \\
10 & 9.7 & 3.5 & $6 \mathrm{~h}$ \\
11 & -1.8 & 10.1 & $18 \mathrm{~h}$ \\
12 & -9.6 & -3.6 & $18 \mathrm{~h}$ \\
13 & 1.7 & -10.1 & $6 \mathrm{~h}$ \\
\hline
\end{tabular}

Table 3. Coordinates of and local time at the observed points on the planetary disk in 2005. Celestial offsets.

\begin{tabular}{cccc}
\hline \hline Position number & \multicolumn{2}{c}{ Offsets ["] } & Approximate local time \\
& East-West & North-South \\
\hline 1 & 0.0 & 0.0 & $12 \mathrm{~h}$ \\
2 & -3.2 & 4.0 & $12 \mathrm{~h}$ \\
3 & -6.3 & 7.9 & $12 \mathrm{~h}$ \\
4 & -4.0 & -3.2 & $14 \mathrm{~h}$ \\
5 & -7.9 & -6.3 & $18 \mathrm{~h}$ \\
6 & 3.2 & -4.0 & $12 \mathrm{~h}$ \\
7 & 6.3 & -7.9 & $12 \mathrm{~h}$ \\
8 & 4.0 & 3.2 & $10 \mathrm{~h}$ \\
9 & 7.9 & 6.3 & $6 \mathrm{~h}$ \\
10 & 1.1 & 10.0 & $8 \mathrm{~h}$ \\
11 & -10.0 & 1.1 & $18 \mathrm{~h}$ \\
12 & -1.1 & -10.0 & $18 \mathrm{~h}$ \\
13 & 10.0 & -1.1 & $7 \mathrm{~h}$ \\
\hline
\end{tabular}

the map, from the beginning to the end of each run and even from one night to another (13-17 June 2001). Thus, it allows us to average the spectra of a given observed point of the map in order to improve the signal-to-noise ratio.

The SEP spectra (position 1 in Fig. 1, local time: midday) were used to retrieve the atmospheric thermal profile. Given the size of the beam, the latitudinal variations of the thermal profile are averaged and the observed thermal profile can be considered as a mean thermal profile for the whole planetary disk. The thermal profiles of the 12 other points could also be retrieved with our method, but it was not the primary goal of this work. Moreover, the beam size being half the size of the planet, positions 2, 4, 6 and 8 would result in comparable profiles. The 12 remaining points were used to retrieve wind velocities. The coordinates of the 13 points on the Martian map were determined by using the SEP coordinates and supposing that the observations were all carried out at the median date of both sets. Thus, the 


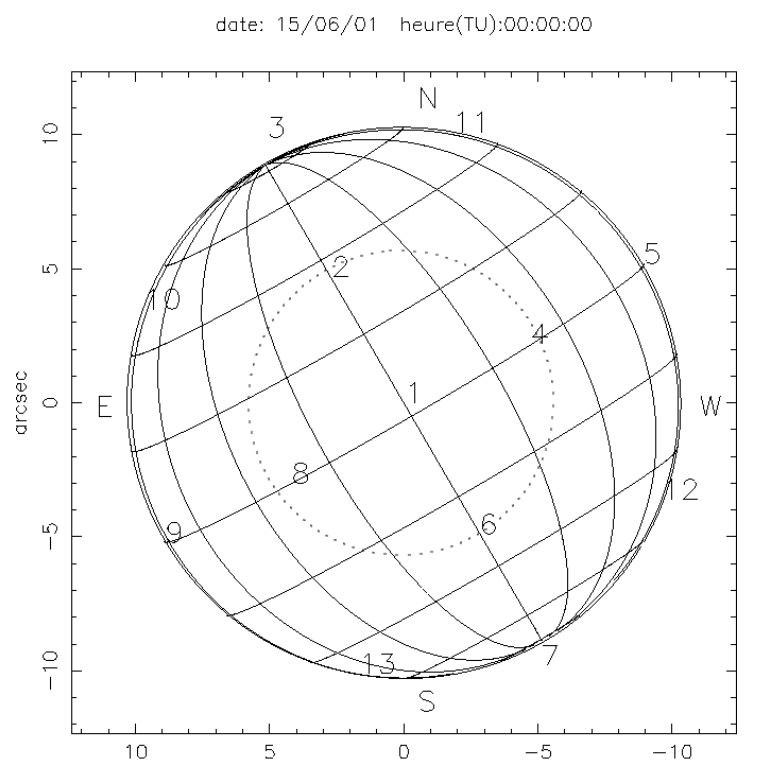

(a)

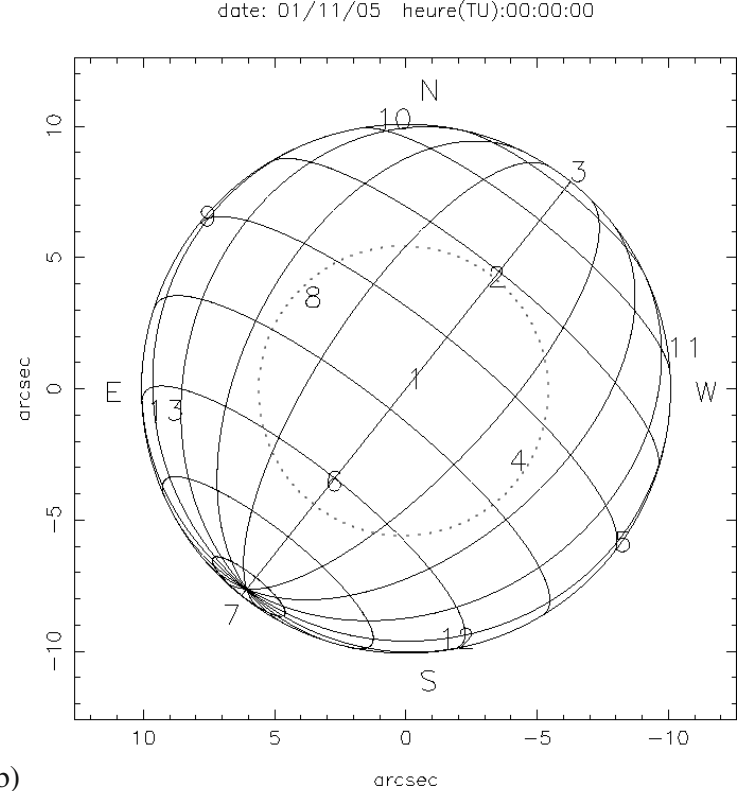

(b)

Fig. 1. Geometry of the observations in a) 2001 and b) 2005. Positions of observed points are given in Tables 2 and 3. Their position on the disks are only approximative. The field of view (full width at half maximum of the beam) at $230 \mathrm{GHz}$ is plotted in dashed lines for position 1. Celestial east/west directions.

velocities are averaged over $80^{\circ}$ in longitude, due to the rotation of the planet during each observing run. Having these coordinates enables us to retrieve the predicted wind velocities with the MCD model.

Two receivers at 1 and 3-mm wavelength were used simultaneously during each run in order to observe the ${ }^{12} \mathrm{CO}$ and ${ }^{13} \mathrm{CO} J=1-0$ and $J=2-1$ lines. The observations were carried out in a singleside band (SSB) mode with a band rejection factor of 0.01 on the $115 \mathrm{GHz}$ band and 0.05 on the $230 \mathrm{GHz}$ band. The system temperatures and atmospheric opacities are given in Table 4 . The opacity is higher at $115-\mathrm{GHz}$ than at $230-\mathrm{GHz}$ because the $115-\mathrm{GHz}$ band is in the vicinity of a strong $\mathrm{O}_{2}$ terrestrial absorption band (at 118-GHz). The filterbanks were set in two modes: a $1 \mathrm{MHz}$ low resolution mode in order to have the total line shape and contrast for the ${ }^{12} \mathrm{CO}(1-0)$ and ${ }^{12} \mathrm{CO}(2-1)$ and
Table 4. Observational parameters in 2001 and 2005.

\begin{tabular}{cccc}
\hline \hline Receivers & $3 \mathrm{~mm}$ & \multicolumn{2}{c}{$1 \mathrm{~mm}$} \\
\hline$T_{\text {sys }}(2001)[\mathrm{K}]$ & $400-420$ & $250-350$ \\
$\tau_{\text {zenith }}(2001)$ & 0.25 & 0.04 \\
$T_{\text {sys }}(2005)[\mathrm{K}]$ & $240-260$ & $220-240$ \\
$\tau_{\text {zenith }}(2005)$ & 0.32 & \multicolumn{2}{c}{0.09} \\
\hline Line & ${ }^{12} \mathrm{CO}(1-0)$ & ${ }^{13} \mathrm{CO}(2-1)$ & ${ }^{12} \mathrm{CO}(2-1)$ \\
\hline Frequency $[\mathrm{GHz}]$ & 115.271 & 220.399 & 230.538 \\
Beam size $(F W H M)[\operatorname{arcsec}]$ & 21.5 & 11.1 & 10.6 \\
Low resolution $[\mathrm{MHz}]$ & 1.0 & not used & 1.0 \\
Bandwidth $[\mathrm{MHz}]$ & 256 & not used & 256 \\
High res. $(2001)[\mathrm{kHz}]$ & 39.0 & 78.0 & 39.0 \\
High res. $(2005)[\mathrm{kHz}]$ & 26.4 & 26.4 & 26.4 \\
Bandwidth $[\mathrm{MHz}]$ & 17 & 17 & 17 \\
\hline
\end{tabular}

a high resolution mode to have the line cores of each ${ }^{12} \mathrm{CO}$ and ${ }^{13} \mathrm{CO}$ line. The high resolution lines are used to retrieve the atmospheric thermal profile (at the SEP location) and the wind velocities (on the other locations on the disk). The low resolution spectra are used to retrieve the contrast between $T(z=0 \mathrm{~km})$ and $T_{\text {surf. }}$ Observed lines, beam sizes, and spectral resolutions are displayed in Table 4 . We used the wobbler switching technique, with a $0.5 \mathrm{~Hz}$ frequency and an amplitude of 80 arcsec.

The pointing accuracy was about $1-2$ arcsec but it was not a limitation to our analysis of the planetary mean thermal profile, because the effect of such offsets on the SEP spectra is not significant. Moreover, all spectra are interpreted in terms of lineto-continuum ratio. Ripples (long and short periods) appeared to be the most interfering features on the observed spectra. They were removed by fitting baselines of order 1 to 4 , depending on the $\mathrm{CO}$ line, on the low spectral resolution spectra, for the thermal profile study. All the high resolution spectra were only symmetrised to average the effect of long period ripples. For the wind velocity study, we were only interested in the Doppler shift value, not on the global line shape. In order to fit the core center with a Gaussian curve in a proper way, baselines of higher order (2-8) were used when deriving wind velocities to remove the far wings $(\Delta v>6-\mathrm{MHz}$ at $230-\mathrm{GHz})$. The removed baselines were selected in order to not change the line core.

All CO lines were detected with high signal-to-noise ratios, that is $\simeq 30$ for the ${ }^{12} \mathrm{CO}$ lines and $\simeq 10$ for the ${ }^{13} \mathrm{CO}(2-1)$ line. The ${ }^{13} \mathrm{CO}(1-0)$ weak emission was not used for analysis because of a faint signal-to-noise ratio and strong ripples. A weak absorption feature appears on the blue wing of the ${ }^{12} \mathrm{CO}$ line in the 2001 data (see Fig. 2). This was thought to be the signature of mesospheric CO from the Earth. These terrestrial lines appear sometimes because of the rapid variation of the atmospheric opacity. In this case, the frequency shift is greater by a factor of $\sim 2$ than a shift due to the Earth-Mars relative velocity $(1.5 \mathrm{MHz}$ at $230 \mathrm{GHz}$ ). So, these features might be ripple subtraction remnants. They do not interfere with the fitting of the lines or the wind velocity derivation. The terrestrial $\mathrm{CO}$ absorption does not appear in the 2005 data because the observations were carried out only 2 days after the opposition. So the terrestrial mesospheric CO absorption is not shifted enough from the line center $(0.3 \mathrm{MHz}$ at $230 \mathrm{GHz})$. This adds an uncertainty, which should be low, on the line contrasts that were measured. So, for the thermal profile derivation (not for the wind velocity derivation), all lines were symmetrised to average the amplitude of the remaining ripple features and to improve the signal-to-noise ratio. For the 2005 wind velocity derival, a problem could be that a faint terrestrial CO absorption near the line core could slightly shift it. 

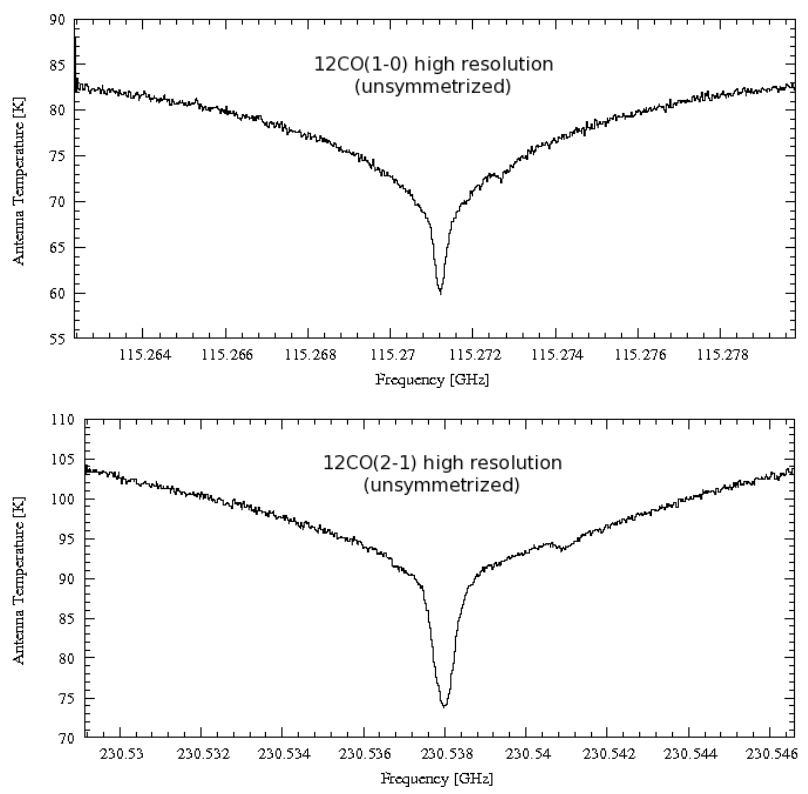

Fig. 2. Antenna temperature spectra of the ${ }^{12} \mathrm{CO}(1-0)$ and $(2-1)$ lines in 2001 for position 1 with the high spectral resolution mode. The absorption caused by terrestrial mesospheric $\mathrm{CO}$ can be seen at $1.5 \mathrm{MHz}$ on the blue wing of the line center of the (1-0) line and $3 \mathrm{MHz}$ on the blue wing of the line center of the $(2-1)$ line.

From the data, there is no evidence of a systematic shift (see shift measurements on position 1 for ${ }^{12} \mathrm{CO}$ and ${ }^{13} \mathrm{CO}$ in Table 11$)$, so this effect (if there is one) cannot be calibrated.

\section{Mean thermal profiles}

\subsection{Radiative transfer model}

We used a line-by-line 1D non-scattering radiative transfer model to compute the synthetic spectrum of Mars in the millimeter range, by solving the radiative transfer equation. Spectra are computed on a regular square grid before averaging them (see below). The model takes into account the spherical geometry so that the limb contributions are naturally accounted for. The atmosphere is divided into $1 \mathrm{~km}$ high levels for altitudes ranging from $0 \mathrm{~km}$ to $120 \mathrm{~km}$. Assuming local thermal equilibrium (LTE), the specific intensity $I_{v}\left[\mathrm{~W} \mathrm{~m}^{-2} \mathrm{sr}^{-1} \mathrm{~Hz}^{-1}\right]$ on a given line-of-sight is:

$I_{v}=\left(I_{v}\right)_{\text {surf }} \mathrm{e}^{-\left(\tau_{v}\right)_{\text {surf }}}+\int_{0}^{\left(\tau_{v}\right)_{\text {surf }}} B_{v}(T(z)) \mathrm{e}^{-\tau_{v}} \mathrm{~d} \tau_{v}$

where $\left(I_{v}\right)_{\text {surf }}$ is the specific intensity emitted by the surface, $\tau_{v}$ is the optical depth, $\left(\tau_{v}\right)_{\text {surf }}$ is the optical depth at the surface level on the considered line-of-sight, $B_{v}(T)$ is the Planck function and $T(z)$ is the atmospheric vertical thermal profile. The specific intensity $\left(I_{v}\right)_{\text {surf }}$ is the grey body radiation of the surface of the planet. It can be written:

$$
\left(I_{v}\right)_{\text {surf }}=\epsilon_{\text {surf }} B_{v}\left(T_{\text {surf }}\right)
$$

where $\epsilon_{\text {surf }}$ is the emissivity of the surface and $T_{\text {surf }}$ is the surface temperature. The value of these parameters is discussed in Sect. 3.1.1. In the case of a line-of-sight that points towards the limb, the specific intensity is obtained from:

$I_{v}=\int_{0}^{\tau_{v}(L)} B_{v}(T(z)) \mathrm{e}^{-\tau_{v}} \mathrm{~d} \tau_{v}$ where $L$ is the total thickness of the atmosphere along the considered line-of-sight. The optical depth is related to the opacity of the atmosphere, which is due to $\mathrm{CO}$ absorption. We assumed all the $\mathrm{CO}$ lines to be Voigt-shaped. Scattering by the dust is neglected because of the small size of the particules $(\sim 1 \mu \mathrm{m}$, Chassefiere et al. 1992) compared to the observed wavelengths.

Specific intensity $\left(I_{v}\right)$ spectra are calculated on a square grid. The final spectra are obtained by averaging the spectra with appropriate weights and convolving with the antenna pattern (assumed to be a 2D Gaussian function). Finally, the $I_{v}$ spectrum is converted into brightness temperature spectra, after taking the filling factor of the antenna into account:

$$
T_{\mathrm{b}}(v)=\frac{h v}{k_{B}}\left[\ln \left(1+\frac{2 h v^{3}}{c^{2}} \frac{1}{I_{v}}\right)\right]^{-1} .
$$

\subsubsection{Surface parameters}

The aim of the data reduction is to retrieve the atmospheric mean thermal profile. The model has several free parameters. Therefore we need to restrain the free parameters as much as possible, before computing the lines. At the surface level, we have 2 parameters, which are the surface pressure and the surface temperature.

The mean surface pressure $p_{\text {surf }}$ values used in our computations are derived from MCD predictions. The value of $p_{\text {surf }}$ depends on the location of the observed site, the time of the observation and the climate scenario chosen (see details in Sect. 3.3). The planetary disk is partitioned into a $61 \times 61$ regular square grid $^{1}$, where the surface pressure is known from MCD predictions. These values are convolved with the antenna pattern in order to have the mean value $p_{\text {surf }}$. Because the antenna pattern width depends on the frequency of the observed line, the values of $p_{\text {surf }}$ depends on the frequency. The values we use for our computations are the $230 \mathrm{GHz}$ values, because the (2-1) line will be the line used to retrieve the thermal profile:

$p_{\text {surf }}(2001)=5.4$ mbar

$p_{\text {surf }}(2005)=6.7$ mbar.

The surface emissivity $\epsilon_{\text {surf }}$ is computed from the Fresnel reflectivity coefficient $R_{\mathrm{F}}$ :

$\epsilon_{\text {surf }}=1-R_{\mathrm{F}}$.

$R_{\mathrm{F}}$ is obtained by averaging the Fresnel reflectivity coefficients for parallel and perpendicular polarizations:

$R_{\mathrm{F}}=\frac{1}{2}\left(\frac{\tan ^{2}\left(\theta_{\mathrm{i}}-\theta_{\mathrm{t}}\right)}{\tan ^{2}\left(\theta_{\mathrm{i}}+\theta_{\mathrm{t}}\right)}+\frac{\sin ^{2}\left(\theta_{\mathrm{i}}-\theta_{\mathrm{t}}\right)}{\sin ^{2}\left(\theta_{\mathrm{i}}+\theta_{\mathrm{t}}\right)}\right)$

where $\theta_{\mathrm{i}}$ is the angle of incidence of the radiation on the surface and $\theta_{\mathrm{t}}$ the transmission angle. The relationship between $\theta_{\mathrm{i}}$ and $\theta_{\mathrm{t}}$ is given by the Snell-Descartes law:

$\sin \left(\theta_{\mathrm{t}}\right)=\frac{\sin \left(\theta_{\mathrm{i}}\right)}{\sqrt{\epsilon}}$

where $\epsilon=2.5$ is the assumed dielectric constant of the surface (Clancy et al. 1983). The disk-averaged value of $\epsilon_{\text {surf }}$ is 0.90 .

\footnotetext{
1 We have a grid with 30 points on each side of the SEP. The value of 30 was chosen after numerical tests, in order to have a good convergence of the results.
} 
Table 5. Mean surface temperature values derived from the 2001 and 2005 observations, after convolution by the antenna beam.

\begin{tabular}{ccc}
\hline \hline Frequency $[\mathrm{GHz}]$ & 115 & 230 \\
\hline Beam width $(F W H M)[\operatorname{arcsec}]$ & 21.5 & 11.6 \\
$\left\langle T_{\text {surf }}\right\rangle_{2001}[\mathrm{~K}]$ & 229 & 249 \\
$\left\langle T_{\text {surf }}\right\rangle_{2005}[\mathrm{~K}]$ & 226 & 246 \\
\hline
\end{tabular}

Table 6. Spectroscopic parameters of the observed CO lines. Parameter $v_{0}$ is given in GHz, $I_{0}$ in $\left[\log \left(\mathrm{nm}^{2} \mathrm{MHz}^{-1}\right)\right]$ at $T=300 \mathrm{~K}$ and $E_{0}$ in $\left[\mathrm{cm}^{-1}\right]$.

\begin{tabular}{cccc}
\hline \hline Parameters & $\mathrm{CO}(1-0)$ & $\mathrm{CO}(2-1)$ & ${ }^{13} \mathrm{CO}(2-1)$ \\
\hline$v_{0}$ & 115.2712018 & 230.5380000 & 220.3986765 \\
$I_{0}$ & -5.0105 & -4.1197 & -4.1749 \\
$E_{0}$ & 0.0 & 3.8450 & 3.6759 \\
\hline
\end{tabular}

Because no absolute calibration was performed, all spectra from 2001 and 2005 are discussed in terms of line-to-continuum ratios and physical surface temperature cannot be derived. Because we want to compare our observations to MCD predictions and because the MCD gives good predictions at low altitudes (Forget, private communication), we choose to take the temperature predicted at $10 \mathrm{~km}$ altitude as a reference for the derivation of the thermal profiles from the observations. So, the temperature profile above and below $10 \mathrm{~km}$ as well as the surface temperature are determined with respect to this fixed value (see Sect. 3.2). Then, we only need a simple model for the surface temperature. We use a very simplified modelling of the surface physical temperature:

$T_{\text {surf }}=T_{0}+T_{1} \cos (2 \alpha)$.

The value of $\alpha$ varies from 0 for the SEP to $\pi / 2$ for a limb position. This model assumes a circular symmetry around the SEP. From thermal emission observations and models (Mellon et al. 2000), it is known that the maximum temperature occurs after local noon. However, due to our relatively low spatial resolution (half of the planetary disk at 230-GHz), we only need to obtain a good approximation of the MCD disk-averaged surface temperature. The values of $T_{1}$ and $T_{0}$ are adjusted in order to obtain a good fit of the far wings of the observed lines and to be close to MCD disk-averaged predictions. The antenna beam convolved values of $T_{\text {surf }}$ are given in Table 5. These values are consistent with Smith (2004) measurements (see their Fig. 11).

\subsubsection{Spectroscopic parameters}

All spectroscopic parameters were taken from the JPL catalog (Pickett et al. 1998). The values taken for the computations are given in Table 6. Parameter $v_{0}$ is the central frequency of the line, $I_{0}$ is the line intensity at $T=300 \mathrm{~K}$ and $E_{0}$ is the energy level of the lower state of the transition. The Lorentzian parameters $\gamma$ and $x$ for ${ }^{12} \mathrm{CO}$ were taken from Varanasi (1975):

$\gamma_{0}=0.11 \mathrm{~cm}^{-1} \mathrm{~atm}^{-1}$

$x=0.75$.

We assume that these parameters can also be taken for ${ }^{13} \mathrm{CO}$. The $\frac{{ }^{12} \mathrm{CO}}{{ }^{13} \mathrm{CO}}$ isotopic ratio is set to 89 (Owen et al. 1977), which is almost the terrestrial value (90).

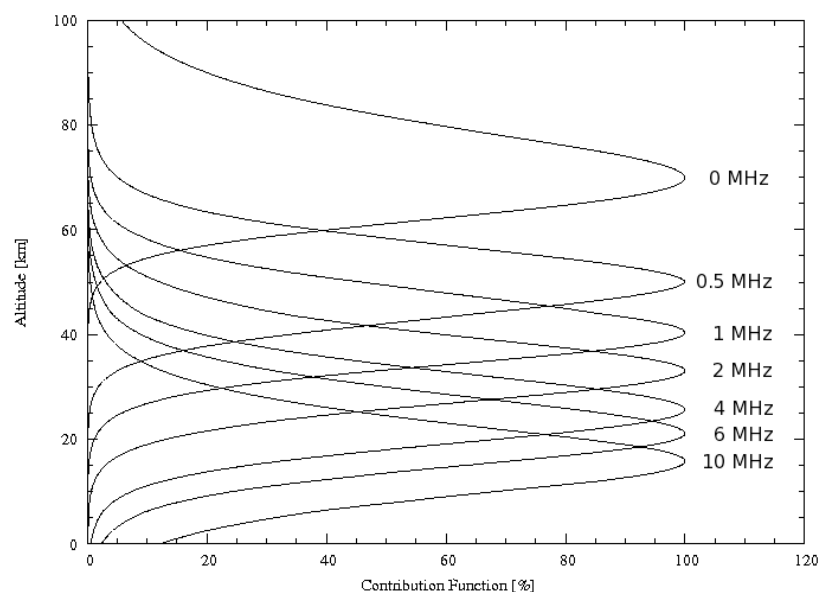

Fig. 3. Contribution functions for the ${ }^{12} \mathrm{CO}(2-1)$ line in 2005. From the highest peak to the lowest one, the frequencies at which the functions are computed are: $0,0.5,1,2,4,6$ and $10 \mathrm{MHz}$ from the line center. These functions show that it is possible to retrieve the thermal profile between 15 and $70 \mathrm{~km}$ from the high resolution ${ }^{12} \mathrm{CO}(2-1)$ line. The ${ }^{12} \mathrm{CO}(1-0)$ line gives similar results. The gap from the surface to $15 \mathrm{~km}$ is filled in with the low resolution ${ }^{12} \mathrm{CO}(2-1)$ spectrum because it gives access to the missing part of the spectrum, from $10 \mathrm{MHz}$ from the line center to the continuum.

\subsection{CO volume mixing ratio and mean thermal profile inversion}

As was mentioned previously, the atmospheric temperature is fixed at the $10 \mathrm{~km}$ altitude MCD prediction value. Because we observe an optically thin line $\left({ }^{13} \mathrm{CO}(2-1)\right)$ and optically thick lines $\left({ }^{12} \mathrm{CO}(1-0)\right.$ and $\left.(2-1)\right)$, we can simultaneously retrieve $q_{\mathrm{CO}}$ and $T(z)$. The procedure used to derive $q_{\mathrm{CO}}, T_{\text {surf }}$ and $T(z)$ is as follows:

- MCD predictions for $q_{\mathrm{CO}}, T_{\text {surf }}$ and $T(z)$ are used to compute the $\mathrm{CO}$ lines. The value of $q_{\mathrm{CO}}$ is assumed to be constant with altitude;

- the surface temperature and the temperature of the first atmospheric levels are adjusted to match the far wings of the ${ }^{12} \mathrm{CO}(1-0)$ and ${ }^{12} \mathrm{CO}(2-1)$ lines. The parameter $q_{\mathrm{CO}}$ is adjusted with the ${ }^{13} \mathrm{CO}(2-1)$ line core, as this line is the only optically thin line $(\tau=0.25$ at the zenith and at the central frequency) that has been observed;

- the high resolution ${ }^{12} \mathrm{CO}(1-0)$ and ${ }^{12} \mathrm{CO}(2-1)$ spectra allow the inversion of the thermal profile above $10 \mathrm{~km}$ altitude (see contribution functions in Fig. 3), as the lines are optically thick ( $\tau=23.5$ at the zenith and at the core center);

- repeat the two previous steps until a good match is reached for the whole set of lines.

For all datasets, the $\mathrm{CO}$ mixing ratio was set to $8.5 \times 10^{-4}$, constant with altitude. We note that some models show an increase of the CO mixing ratio above an altitude of $40 \mathrm{~km}$ because of transport (Theodore et al. 1993; Nair et al. 1994). We have chosen to set this parameter to a constant value in order to simplify the problem, as the only line sensitive to $q_{\mathrm{CO}}$ is the ${ }^{13} \mathrm{CO}(2-1)$ line (optically thin). It appears that considering a variable $q_{\text {CO }}$ profile gives slightly better fits to the ${ }^{13} \mathrm{CO}(2-1)$ line, as shown in Fig. 7 in Theodore et al. (1993). The contribution function of the ${ }^{13} \mathrm{CO}(2-1)$ line peaks at $40 \mathrm{~km}$ (see Fig. 4). So this line is not too sensitive to a slow increase of $q_{\text {CO }}$ above $50 \mathrm{~km}$. The Theodore et al. (1993) model has a homopause level located at 50-55 km and shows that $q_{\mathrm{CO}}$ increases up to $13.0 \times 10^{-4}$ at $60 \mathrm{~km}$, where the contribution function of 


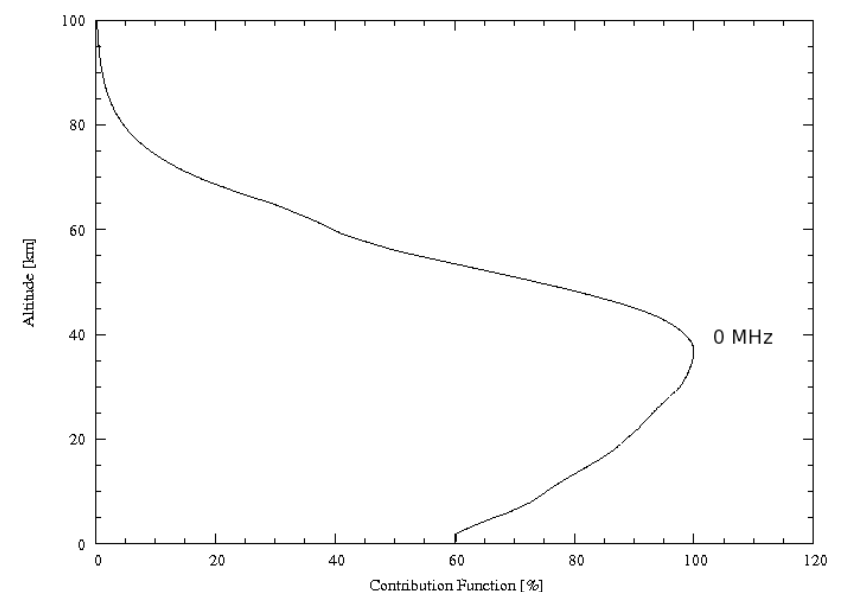

Fig. 4. Contribution function for the central frequency of the ${ }^{13} \mathrm{CO}(2-1)$ line in 2001.

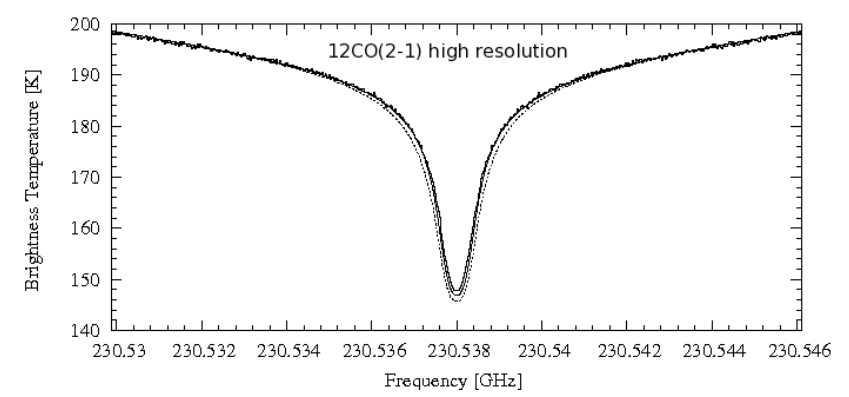

Fig. 5. ${ }^{12} \mathrm{CO}(2-1)$ high resolution line in 2005 fitted with the best fit model (solid line). The dotted model corresponds to an increase of $q_{\mathrm{CO}}$ from $8.5 \times 10^{-4}$ to $13.0 \times 10^{-4}$ above $50 \mathrm{~km}$ (homopause level).

the ${ }^{12} \mathrm{CO}$ line peaks. Nevertheless, since these lines are optically thick, they show no significant change in the line contrast. The width of the line is only slightly affected by this effect (see Fig. 5). Indeed, the ${ }^{12} \mathrm{CO}$ line core shapes at less than $1 \mathrm{MHz}$ from the line center are formed above $40 \mathrm{~km}$. So, fitting the line with such a $\mathrm{CO}$ mixing ratio profile would lead to an increase of the atmospheric temperature by only a few $\mathrm{K}$ above $40 \mathrm{~km}$.

The thermal profile is computed from 0 to $18 \mathrm{~km}$ with a $2 \mathrm{~km}$ step, from 22 to $38 \mathrm{~km}$ with a $4 \mathrm{~km}$ step and from 44 to $116 \mathrm{~km}$ with a $6 \mathrm{~km}$ step. For each point, the temperature is adjusted in order to fit the ${ }^{12} \mathrm{CO}$ and the ${ }^{13} \mathrm{CO}$ high resolution lines. The low atmosphere temperature profile is oversampled with regard to our vertical resolution (gas scale height), so the profile is smoothed. We also add 2 values at $59 \mathrm{~km}$ and $65 \mathrm{~km}$ in order to be able to satisfy the rapid changes in the line core shape. Indeed, the ${ }^{12} \mathrm{CO}$ line sets from 2001 and 2005 show an important increase of the absorption feature, especially in the ${ }^{12} \mathrm{CO}(2-1)$ lines. This effect was observed with the JCMT antenna by Clancy et al. (2006) on 4 November 2005 in the $\mathrm{CO}(3-2)$ line. It is even more significant in the 2001 data (see Fig. 2). Since the ${ }^{12} \mathrm{CO}$ lines are optically thick, it implies that there is a strong and sudden decrease of the temperature for altitudes above $40-50 \mathrm{~km}$, according to contribution function analysis.

\subsection{Using the Mars Climate Database to compute predicted spectra - comparison with observational data}

The Mars Climate Database (MCD) numerical tool (Lewis et al. 1999; Forget et al. 2006) can be used to provide a large number
Table 7. Mean surface temperature given by the MCD for both observation dates and both scenarios (MY24 and Warm) after convolution by the beam pattern at $230 \mathrm{GHz}$.

\begin{tabular}{ccc}
\hline \hline$T_{\text {surf }}[\mathrm{K}]$ & 2001 & 2005 \\
\hline MY24 scenario & 265 & 250 \\
Warm scenario & 261 & 241 \\
\hline
\end{tabular}

of parameters both of the surface and atmosphere over an entire Martian year for a given point on the Martian map. A large panel of different climate databases can be explored with the Fortran subroutine atmemcd (each climate scenario is given a number from 1 to 8$)$. The three different scenarios we have used are number 2 (Mars Year 24), 5 (Dust storm, $\tau=4$ ) and 7 (Warm). The parameters we are interested in are the parameters we defined previously: the surface temperature, the surface pressure, the atmospheric number density and temperature profiles. The $\mathrm{CO}$ mixing ratio as a function of altitude is also given. We start our computations with a mean value of $q_{\mathrm{CO}}=8.5 \times 10^{-4}$ that is consistent with predictions and we set it constant with altitude.

Thus, we have to define the planetocentric points we are interested in. We know the SEP coordinates for all of our observations from the Institut de Mécanique Céleste et de Calcul des Éphémérides (IMCCE) database (http : //www . imcce . fr/ imcce_fr.html). Because the general thermal structure of the atmosphere and surface does not vary too much at a fixed local time, it is possible to average the spectra that correspond to the same local time. As all the spectra we have used correspond to the SEP observations, the local time on the planet is always midday. So, the 5 spectra sets of the 2001 observations are averaged and we take the mean date of the observations to obtain the information we need from the MCD: 15 June 2001 at $0 \mathrm{~h} \mathrm{UTC}$. In 2005, the date we take is 1 November at $0 \mathrm{~h} \mathrm{UTC.}$ For each period, the Martian disk is partitioned in a square grid. The coordinates of each point of the grid are computed in order to obtain a thermal vertical profile by using the atmemcd subroutine. We also have the surface parameters for each point. After convolving by the antenna beam, the mean surface temperatures values given by the MCD predictions are given in Table 7. All profiles are stored and used by the radiative transfer code, in order to obtain a spectrum for each line-of-sight. All these spectra are finally averaged with the appropriate weights to generate the MCD-predicted spectrum for the time of the observations. A spectrum is computed for each observed CO line.

There is an uncertainty in the computation of the temperature vertical profile in the lines of sight that are close to the limbs and poles. Indeed, we have taken the vertical profiles above geographical points when we should have taken the profile in the line-of-sight. The discrepancy is due to the viewing angle which is increasing when going from the disk center to the limbs or poles. The difference between the planetocentric point we use and the one that should be used when we are sounding the limb, for example, is about $740 \mathrm{~km}$ at the top of the atmosphere we are able to sound $(80 \mathrm{~km})$. This means that the difference is about $20 \%$ of the planetary radius. But, the higher the altitude, the more uniform on a large scale the temperature becomes. This is the reason why we choose to use these vertical profiles above the planetocentric points of our map in order not to complicate the computations too much. The uncertainty that comes from this approximation for scenario 2, for example, is about $2-4 \mathrm{~K}$ at $40 \mathrm{~km}$ and $4-6 \mathrm{~K}$ at $60 \mathrm{~km}$ in 2001 and $2-3 \mathrm{~K}$ at $40 \mathrm{~km}$ and $2-4 \mathrm{~K}$ at $60 \mathrm{~km}$ in 2005 for the lines-of-sight that are close to the limb. 
Once we have all the predicted spectra, for each scenario and period of observation (18 spectra; 3 CO lines, 3 scenarios and 2 periods), we use the radiative transfer code to determine the mean thermal profile of the planet that would lead to the predicted features. The thermal profiles can then directly be compared with the mean thermal profiles derived from the observations. This procedure gives us a unique tool to compare a predicted mean thermal profile with the observed one.

Figure 6 shows the comparison between the observations carried out with the 30-m telescope and the MCD prediction for scenarios 2 (MY24), 5 (Dust storm) and 7 (Warm), in terms of relative intensity. The same plots are displayed in Fig. 7 for the 2005 data. They all clearly show that, despite the fact that the data were obtained either a few days before a global dust storm or a few days after a regional dust storm, the Dust storm scenario from the MCD database is not appropriate to compute CO lines with the right line shape in these cases. The MY24 scenario provides better fits to the data. Nevertheless, we can see from both Figs. 6 and 7 that the Warm scenario gives the best basis to retrieve the observed mean thermal profiles from the MCD predictions. The Warm scenario gives good line fits for the 2001 and 2005 data, except in the line core at high resolution. This means that differences between the observed mean thermal profile and the MCD prediction are above $40 \mathrm{~km}$. Finally, the observed mean profiles will be compared to the MY24 and Warm scenario mean profiles.

\subsection{Thermal profile retrieved from the observational data}

The surface temperature parameters of our simplified model are $\left(T_{0}, T_{1}\right)=(235 \mathrm{~K}, 49 \mathrm{~K})$ for the 2001 observations and $\left(T_{0}, T_{1}\right)=(232 \mathrm{~K}, 50 \mathrm{~K})$ for the 2005 observations. The detailed values of the atmospheric temperatures retrieved from the 2001 and 2005 linesets are given in Tables 8 and 9. The profiles are displayed in Fig. 8. The high resolution spectra give us the opportunity to derive a high precision mean thermal profile because the temperature at $10 \mathrm{~km}$ was fixed to the MCD value (reference value). The uncertainty on the temperature values is about $3-5 \mathrm{~K}$.

So, the observations were fitted with the thermal profiles presented in Fig. 8. Both profiles (of 2001 and 2005) give satisfactory fits to all the observed spectra (see Figs. 9 and 10). Considering the ${ }^{12} \mathrm{CO}(1-0)$ line, the first atmospheric layer/surface temperature contrast is well-modeled and permits a good match in the emission far wings of the line either in 2001 or 2005. This is the only line where the beam has a size comparable to the planet ( $\sim 20 \mathrm{arcsec})$ so that we are sensitive to the strong variations of $T_{\text {surf }}$ at the eastern and western limbs and at the northern and southern poles. The ${ }^{13} \mathrm{CO}(2-1)$ line is not wellreproduced with the model. The model gives too broad wings. Such a limit in the modeling of ${ }^{13} \mathrm{CO}$ lines can be seen in several papers (Lellouch et al. 1989; Encrenaz et al. 2001).

When compared to the MCD predictions, several discrepancies can be underlined between the predictions and the observations. The fact that the surface and first layer temperatures from the observed sets are not the same as the one of the predicted sets is not too restrictive, since we have no absolute calibration. Moreover, the subtraction of the ripples from the observations adds a large uncertainty on the contrast between the surface temperature and the temperature of the first atmospheric layers. This is also the reason why our observed mean thermal profile cannot be compared to predictions below the altitude of $10 \mathrm{~km}$.

In the case of the 2001 data, there is a very good match of the prediction of the MY24 and Warm scenarios to the observations

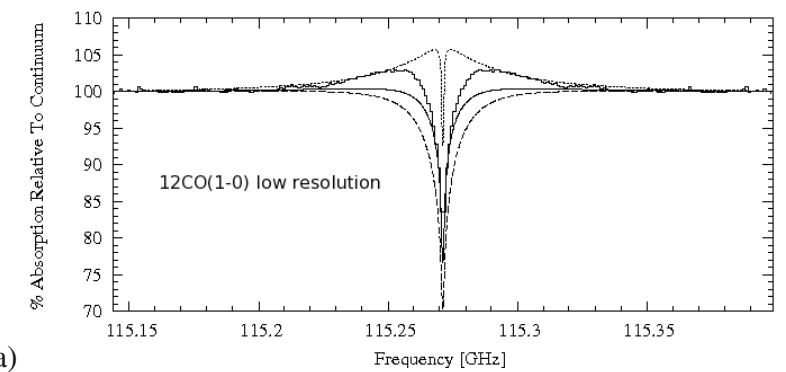

(a)

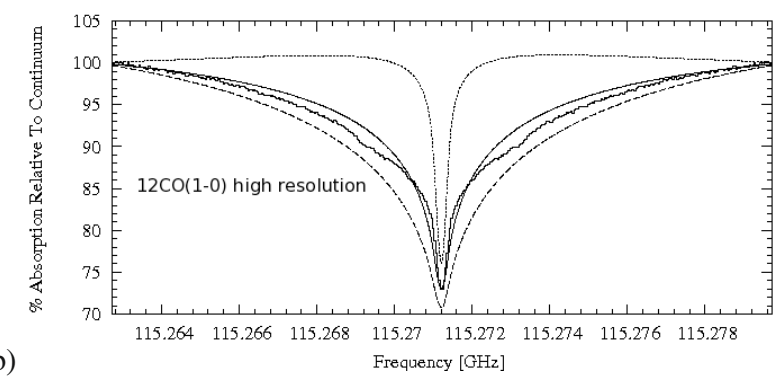

(b)
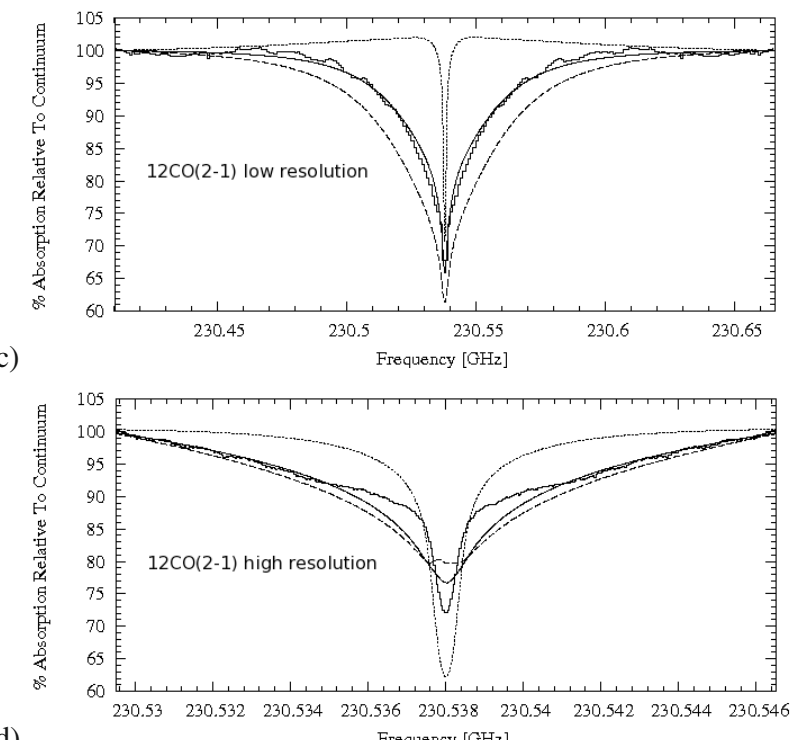

(d)

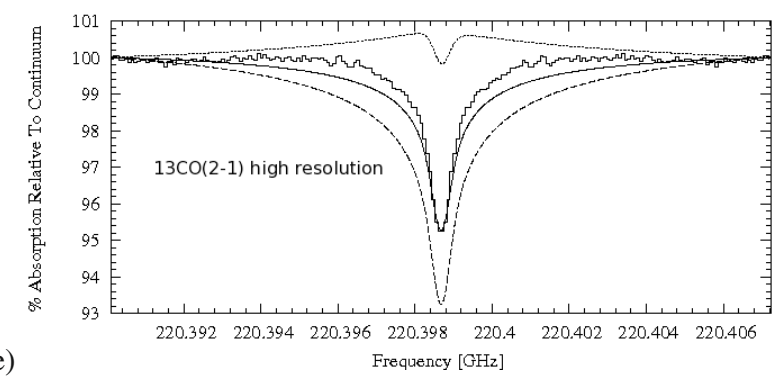

Fig. 6. Relative comparison between the observed spectra of 2001 and MCD predictions (Warm scenario in solid lines, dust storm scenario in dotted lines and MY24 scenario in long-dashed lines) for: ${ }^{12} \mathrm{CO}(1-0)$ a) low and b) high resolution, ${ }^{12} \mathrm{CO}(2-1)$ c) low and d) high resolution, ${ }^{13} \mathrm{CO}(2-1)$ e) high resolution.

from 10 to $30 \mathrm{~km}$. But, there is a major discrepency between 30 and $80 \mathrm{~km}$. From 30 to $60 \mathrm{~km}$, the observed temperatures are warmer than the predicted ones by $10-15 \mathrm{~K}$. And from 60 to $80 \mathrm{~km}$, they are $10 \mathrm{~K}$ lower than the Warm scenario and up to $20 \mathrm{~K}$ lower than the MY24 scenario temperatures. The observed temperature profile shows a sudden and strong decrease, from 


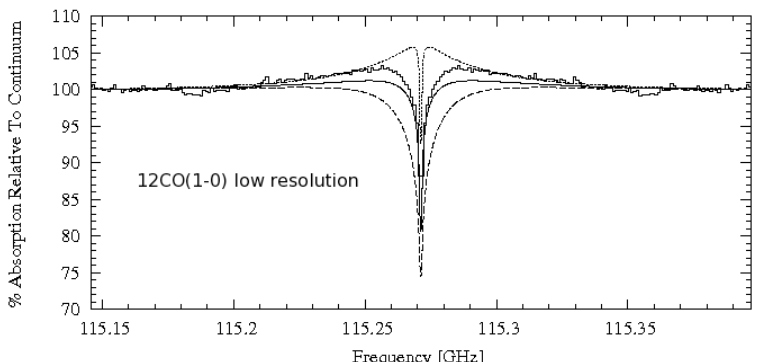

(a)

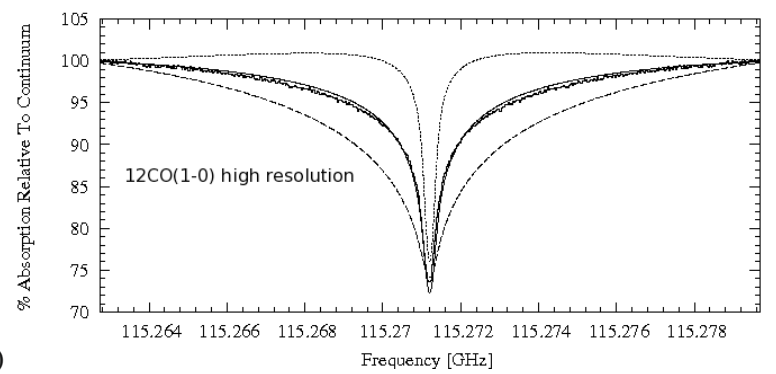

(b)

(c)
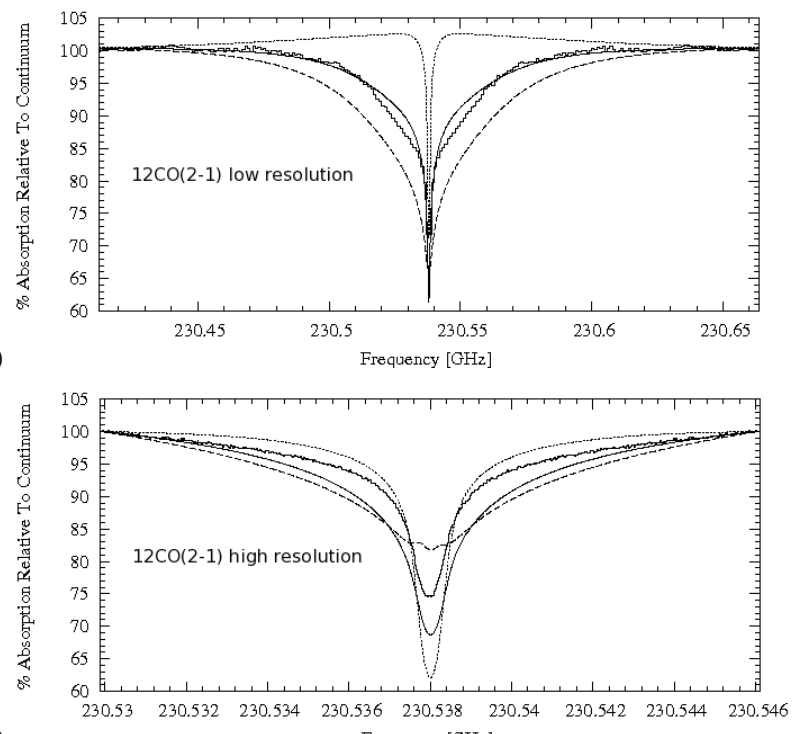

(d)

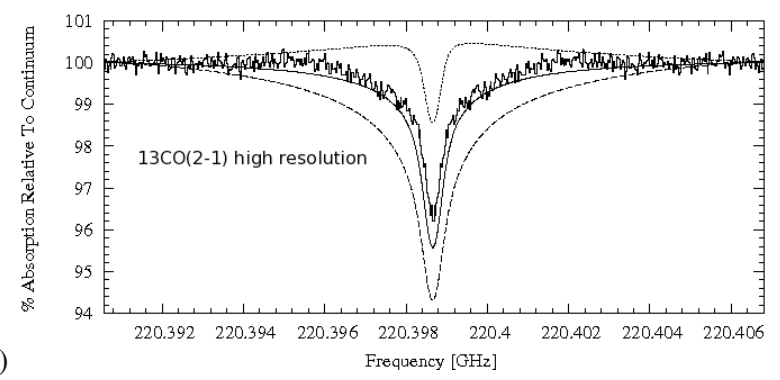

Fig. 7. Same caption as Fig. 6 for 2005.

$168 \mathrm{~K}$ at $56 \mathrm{~km}$ to $140 \mathrm{~K}$ at $68 \mathrm{~km}$. On the other side, the Warm scenario predicts a slow and regular decrease and the MY24 scenario predicts a thermal inversion layer between 50 and $70 \mathrm{~km}$. Finally, we are no longer sensitive to thermal variations above $80 \mathrm{~km}$ (see contribution function in Fig. 3).

The MY24 scenario also predicts a thermal inversion layer between 50 and $70 \mathrm{~km}$ for 2005 . This layer is not seen in the data. The effect of such a layer can be seen in the ${ }^{12} \mathrm{CO}(2-1)$ line, when generated with such a thermal profile. To illustrate this effect, Fig. 11 shows the ${ }^{12} \mathrm{CO}(2-1)$ line computed with the MCD predicted thermal profile of 2005 (MY24 scenario). The line core is very wide compared to the observed line shape. Moreover, two tiny emission features can be seen at $0.3 \mathrm{MHz}$ from the line center. This Doppler shifted emission comes from lines of sight that are in the vicinity of the eastern and western limb. The increase of the total thickness of the atmosphere causes the emission in the center of the line. The thermal profile we derive from the 2005 observations is closer to the Warm scenario thermal profile. There is a very good match from 10 to $30 \mathrm{~km}$. Then, the observed profile is on average $10 \mathrm{~K}$ warmer than the profile that comes from the Warm scenario. Once again, a sudden decrease of the temperature is seen, but at a lower altitude of $(z \simeq 45 \mathrm{~km})$ and with a shallower gradient. This is the reason why the core of the ${ }^{12} \mathrm{CO}(2-1)$ line is not as deep in 2005 as in 2001 .

\subsection{Discussion}

The 2001 observations were carried out a few days before the beginning of the global dust storm that occured that year while the 2005 observations were carried out a few days after a significant regional dust storm (Clancy et al. 2006) over the Terra Arabia region. These are the reasons why three different climate scenarios from the GCM have been tested. The first one corresponds to the standard Mars Year 24 scenario (scenario 2), the second one corresponds to the Dust storm scenario (scenario 5), while the third one corresponds to the Warm scenario (scenario 7). Preliminary comparisons made between the predictions and the observations proved that the Dust storm scenario was not satisfactory. So, the comparison has been made between the observations and the MCD MY24 and Warm scenarios.

In both cases, we observe a significant warming in the range of $30-50 \mathrm{~km}$ (up to $60 \mathrm{~km}$ in 2001) compared to the GCM predictions. It clearly appears that no thermal gradient sign changes were observed in 2001 and 2005 whereas MCD MY24 computations predict a thermal inversion layer between 40 and $60 \mathrm{~km}$. Both thermal profiles have a different behavior from $30 \mathrm{~km}$ up to the top of the observed part of the sounded part of the atmosphere (about $80 \mathrm{~km}$ ) when compared to the MCD predictions.

Our 2005 thermal profile is consistent from 10 to $20 \mathrm{~km}$ with measurements made in the same $L_{\mathrm{S}}$ range $\left(L_{\mathrm{S}}=300-320^{\circ}\right)$ with Mars Global Surveyor TES instrument and the Kitt Peak, Arizona, observatory (Clancy et al. 2000). The profile we retrieve shows significant warming between 20 and $55 \mathrm{~km}$ (up to $20 \mathrm{~K}$ compared to the MY24 scenario and $10 \mathrm{~K}$ compared to the Warm scenario, in 2005). Such a warming can be due to dust loading in this part of the atmosphere because of dust storm activity during this period of the Martian orbit $\left(L_{\mathrm{S}}=310-330^{\circ}\right)$. As was mentionned by Clancy et al. (2006), Mars was observed right after a regional dust storm in 2005 . So, this could be the cause of the observed warming of the middle atmosphere. The higher part of the observed atmosphere $(z \geq 40 \mathrm{~km})$ shows a rapid decrease in temperature. The MY24 scenario temperatures are higher than the observed ones between 50 and $90 \mathrm{~km}$, because of the presence of a thermal inversion layer. The Warm scenario temperature profile is colder than the observed one above $30 \mathrm{~km}$. Figure 11 of Clancy et al. (2006) does not show such a rapid decrease in temperature, but rather a gradual one (see Fig. 12). They observed the ${ }^{12} \mathrm{CO}(3-2)$ and the ${ }^{13} \mathrm{CO}(3-2)$ lines to retrieve the atmospheric thermal profile. The contribution functions of these lines peak respectively at 80 and $40 \mathrm{~km}$ at the core center (see Fig. 13). Therefore, they might have some uncertainty in their retrieval of the 50-70 km layer temperatures. Moreover, a contribution function analysis for the ${ }^{12} \mathrm{CO}(3-2)$ 
Table 8. Mean thermal profiles retrieved from the 2001 observations.

\begin{tabular}{cc|cc|cc}
\hline \hline Altitude $[\mathrm{km}]$ & $T(z)[\mathrm{K}]$ & Altitude $[\mathrm{km}]$ & $T(z)[\mathrm{K}]$ & Altitude $[\mathrm{km}]$ & $T(z)[\mathrm{K}]$ \\
\hline 0 & 250.0 & 22 & 186.5 & 65 & 143.0 \\
2 & 250.0 & 26 & 183.0 & 68 & 140.0 \\
4 & 238.0 & 30 & 178.5 & 74 & 136.0 \\
6 & 225.0 & 34 & 176.0 & 80 & 134.0 \\
8 & 216.0 & 38 & 173.0 & 86 & 133.0 \\
10 & 211.0 & 44 & 170.0 & 92 & 132.0 \\
12 & 206.0 & 50 & 169.0 & 98 & 131.0 \\
14 & 200.5 & 56 & 168.0 & 104 & 131.0 \\
16 & 195.5 & 59 & 163.0 & 110 & 131.0 \\
18 & 191.5 & 62 & 152.0 & 116 & 131.0 \\
\hline
\end{tabular}

Table 9. Mean thermal profiles retrieved from the 2005 observations.

\begin{tabular}{cc|cc|cc}
\hline \hline Altitude $[\mathrm{km}]$ & $T(z)[\mathrm{K}]$ & Altitude $[\mathrm{km}]$ & $T(z)[\mathrm{K}]$ & Altitude $[\mathrm{km}]$ & $T(z)[\mathrm{K}]$ \\
\hline 0 & 234.0 & 22 & 201.5 & 65 & 152.0 \\
2 & 234.0 & 26 & 198.5 & 68 & 151.0 \\
4 & 230.0 & 30 & 195.0 & 74 & 149.0 \\
6 & 228.0 & 34 & 192.0 & 80 & 148.0 \\
8 & 219.0 & 38 & 190.0 & 86 & 147.0 \\
10 & 215.0 & 44 & 187.0 & 92 & 145.0 \\
12 & 209.0 & 50 & 172.0 & 98 & 144.0 \\
14 & 207.0 & 56 & 162.0 & 104 & 143.0 \\
16 & 205.0 & 59 & 156.0 & 110 & 143.0 \\
18 & 203.0 & 62 & 154.0 & 116 & 143.0 \\
\hline \multicolumn{5}{r}{}
\end{tabular}
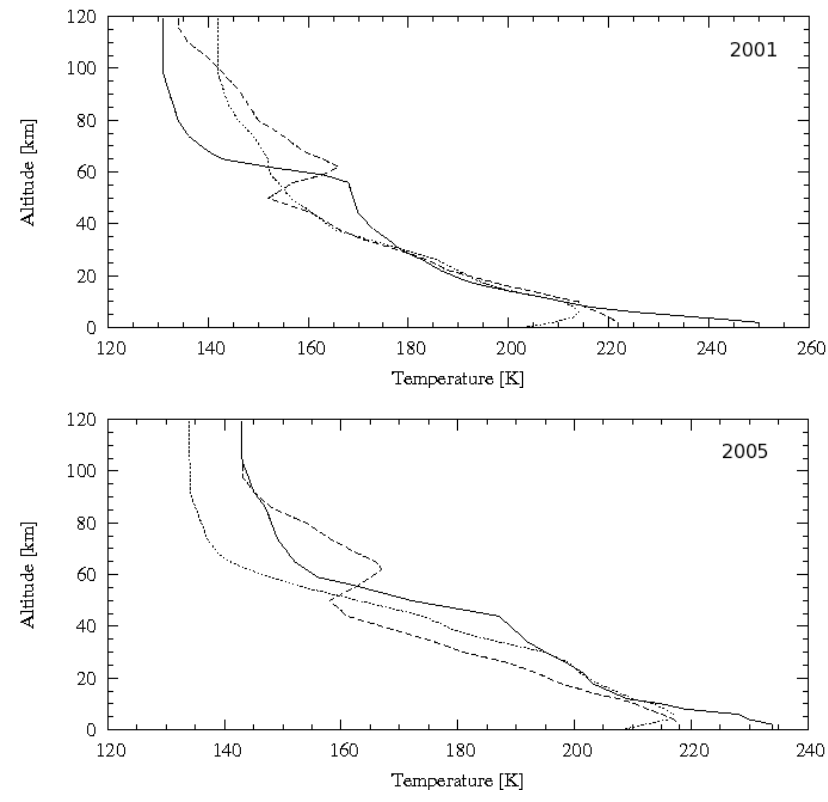

Fig. 8. Mean atmospheric temperature profiles derived from the observations (solid lines) and the MCD predictions in 2001 and 2005. The temperature profile corresponding to the scenario 2 (MY24) and 7 (Warm) are in dashed and dotted lines, respectively.

line shows a dramatic drop in altitude (from 78 to $28 \mathrm{~km}$ ) when going from the line center to $6 \mathrm{MHz}$ away (see Fig. 13). Because the atmospheric levels between 40 and $60 \mathrm{~km}$ correspond to offsets from the central frequency that are lower than $6 \mathrm{MHz}$ and because the change of the line shape (around the line center) occurs at offsets lower than $6 \mathrm{MHz}$, they might not have enough spectral resolution to reproduce more precisely the thermal profile in this altitude zone. However, the line wings permit an effective retrieval below $28 \mathrm{~km}$. If we take our thermal profile to model the ${ }^{12} \mathrm{CO}(3-2)$ line, we obtain too much absorption at the core center compared to their observation (38\% instead of 35\%). As the lines we used to retrieve the thermal profile are not sensitive above $70 \mathrm{~km}$, our retrieval has some uncertainty above this level. If we increase the temperature by a few $\mathrm{K}$ at $80 \mathrm{~km}$ and above, we would have the same absorption level as the Clancy et al. (2006) observation.

The warming observed in the 2001 data is not as intense as the one observed in 2005. The temperature increase occurs from 30 to $60 \mathrm{~km}$ and is about $15 \mathrm{~K}$ in magnitude with respect to the MY24 scenario thermal profile. When compared to the Warm scenario profile, the observed temperature profile is warmer from 30 to $60 \mathrm{~km}$ by $10-15 \mathrm{~K}$. Above $60 \mathrm{~km}$, our thermal profile becomes colder than either the MCD MY24 or Warm scenario temperature profiles. The decrease of the temperature seen from the data is important enough to cause a discrepency of $10-20 \mathrm{~K}$ in the $60-80 \mathrm{~km}$ altitude range. The increase of the temperature between 30 and $60 \mathrm{~km}$ could be the signature of the beginning of the global dust storm that occured from late June until September 2001. Gurwell et al. (2005) observed the ${ }^{13} \mathrm{CO}(5-4)$ line, the $\mathrm{H}_{2}^{16} \mathrm{O}$ and $\mathrm{H}_{2}^{18} \mathrm{O}$ ground states during the global dust storm of 2001 on Mars with the SubmillimeterWave Astronomy Satellite. They retrieved the surface temperature value (12 K uncertainty) and atmospheric temperature $\left(\sim 7 \mathrm{~K}\right.$ uncertainty) at 3 levels $(25,45$ and $66 \mathrm{~km})$ from $L_{\mathrm{S}}=170^{\circ}$ to $L_{\mathrm{S}}=230^{\circ}$. As the SWAS beam was much larger than the planet's size, they retrieved mean values of these parameters. The mean surface temperature value for $L_{\mathrm{S}}=180^{\circ}$ given by our model is $249 \mathrm{~K}$. It is consistent with the Gurwell et al. (2005) value on 21 June 2001 (opposition, $L_{\mathrm{S}}=182^{\circ}$ ), which is $260 \pm 12 \mathrm{~K}$. As the surface temperatures are consistent, it is possible to compare the mean atmospheric temperature values. From their Fig. 3, we see that, at $L_{\mathrm{S}}=180^{\circ}$, they find: $188 \pm 2 \mathrm{~K}$ at $25 \mathrm{~km}, 170 \pm 3 \mathrm{~K}$ at $45 \mathrm{~km}$ and $160 \pm 5 \mathrm{~K}$ at $66 \mathrm{~km}$ (see Fig. 14). The values we derive are quite similar at 25 and $45 \mathrm{~km}$ (182.5 $\pm 5 \mathrm{~K}$ and $169.5 \pm 5 \mathrm{~K}$, respectively). However, we retrieve a different value at $66 \mathrm{~km}$. Our value is $20 \mathrm{~K}$ lower $(140 \pm 5 \mathrm{~K})$. 


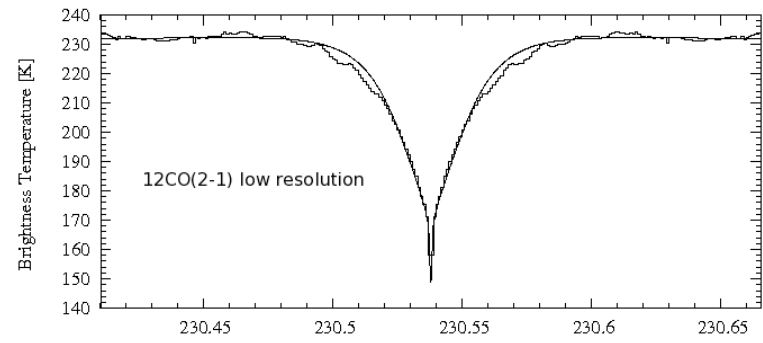

(a)

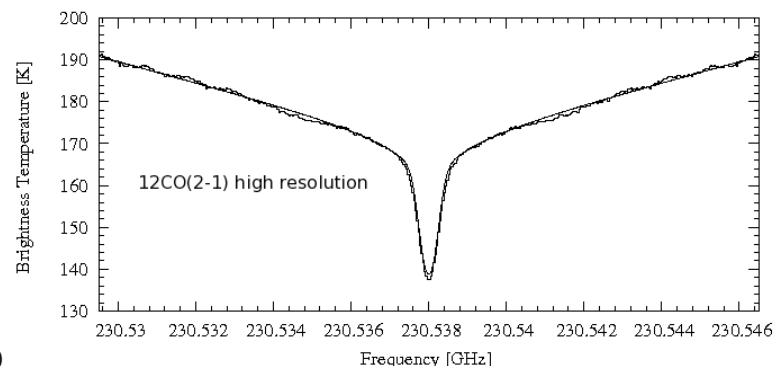

(b)

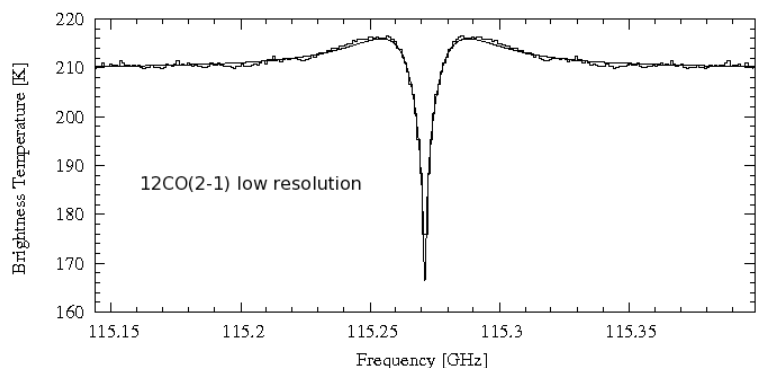

(c)

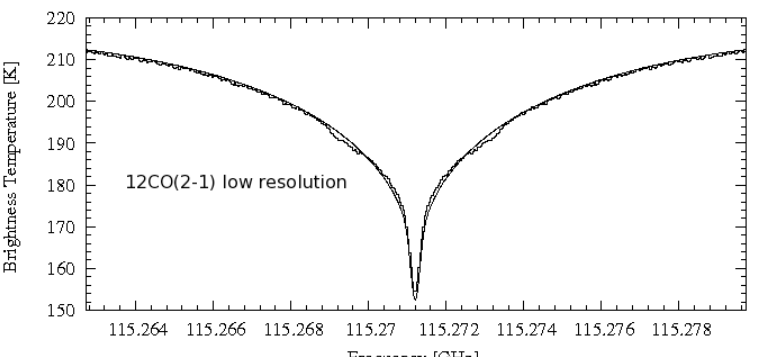

(d)

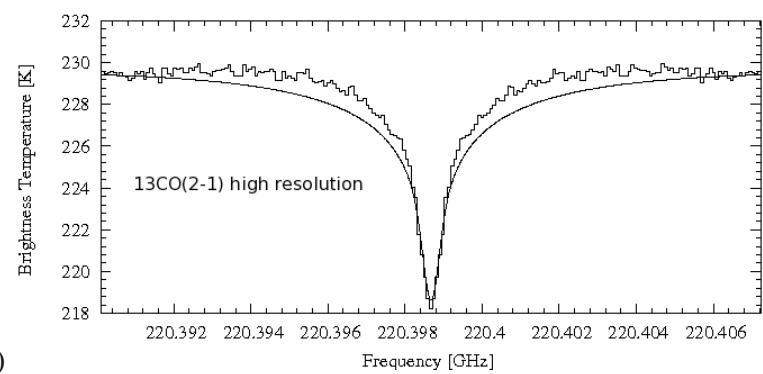

Fig. 9. Best fit model for the 2001 dataset. The mean temperature profile derived from the observations is shown in Fig. 8. The 1-D surface temperature profile used is given in Eq. (10). The CO mixing ratio is $8.5 \times 10^{-4}$. All spectra correspond to disk center observations. a) ${ }^{12} \mathrm{CO}(2-1)$ line with $256 \mathrm{MHz}$ bandwidth and a $1 \mathrm{MHz}$ spectral resolution. b) Same line but with $128 \mathrm{MHz}$ bandwidth and a $39 \mathrm{kHz}$ spectral resolution. c) ${ }^{12} \mathrm{CO}(1-0)$ line with the same parameters as a). d) ${ }^{12} \mathrm{CO}(1-0)$ line with the same parameters as b). d) ${ }^{13} \mathrm{CO}(2-1)$ line with a $78 \mathrm{kHz}$ spectral resolution.

Because the ${ }^{13} \mathrm{CO}(5-4)$ line cannot be used to retrieve temperatures above $40 \mathrm{~km}$ (contribution function study), Gurwell et al. (2005) also used water vapor lines to compute their temperature

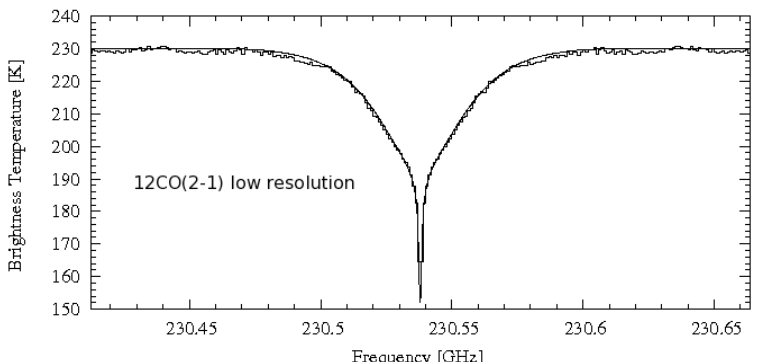

(a)
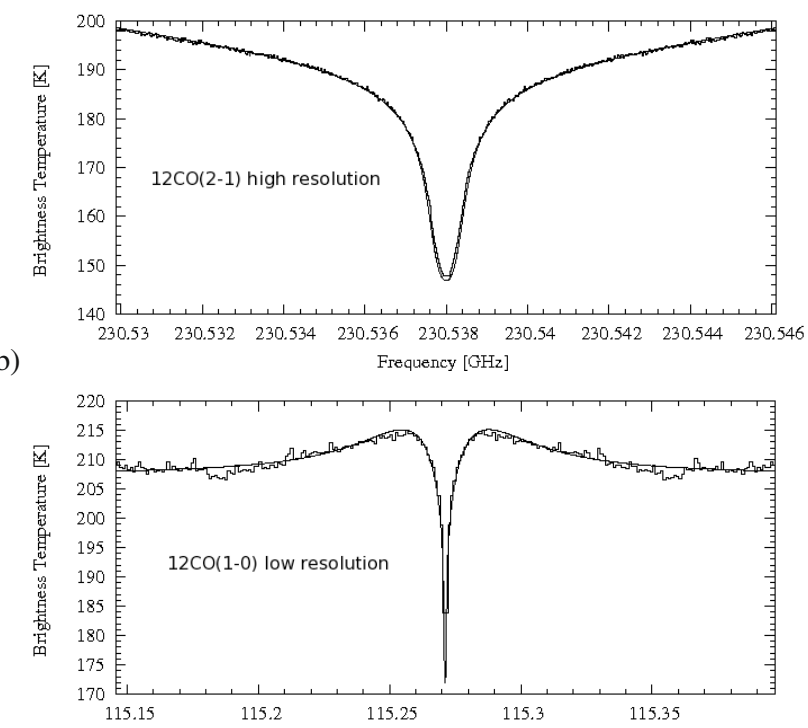

(c)
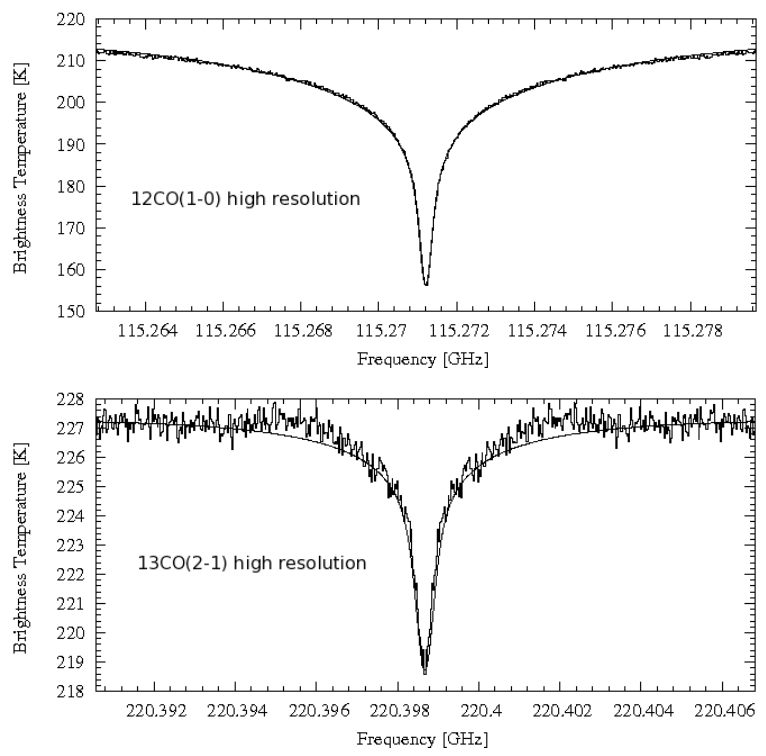

Fig. 10. Same caption as Fig. 9 for the 2005 dataset. Mean temperature profile shown in Fig. 8b. The spectral resolution is $26.4 \mathrm{kHz}$ for the b), d) and e) spectra.

profile above this level. The thermal profile retrieval depends on the assumption that the water vapor profile is only governed by vapor saturation.

Finally, a thick layer which is warmer than predicted by the MCD has been observed in the 10-50 km range in 2001 and in 2005 . In the $50-90 \mathrm{~km}$ range, atmospheric temperatures lower than predicted by the MCD have been observed. These observations tend to confirm results recently obtained from stellar 


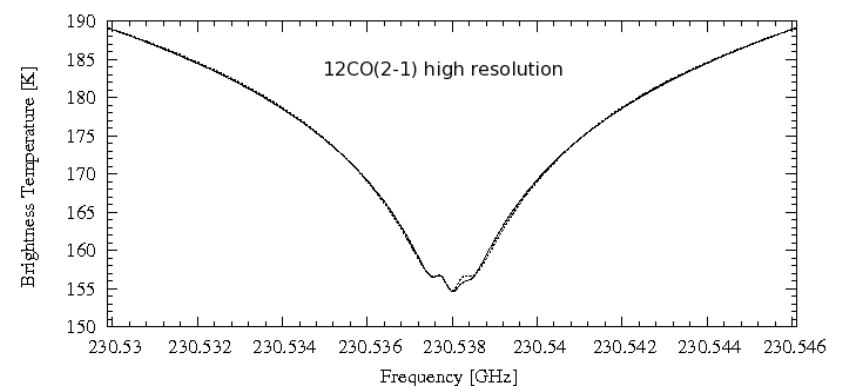

Fig. 11. ${ }^{12} \mathrm{CO}(2-1)$ line computed with the MCD thermal profiles (black lines) taken from the $61 \times 61$ grid of 2005 . The line is fitted with the mean thermal profile model (dashed lines).

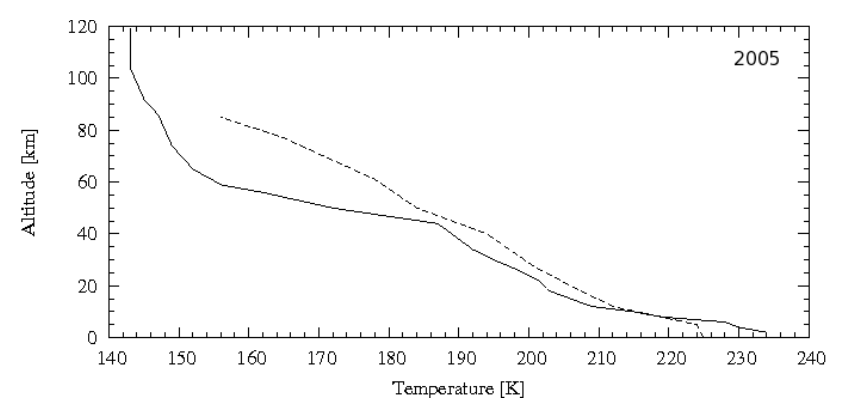

Fig. 12. Atmospheric temperature profile in 2005 derived from the observations (solid lines) and from Clancy et al. (2006) (dashed lines).

occultations with the SPICAM ultraviolet spectrometer aboard Mars Express (Forget et al. 2007).

\section{Mesospheric winds}

\subsection{Wind velocity retrieval}

Wind velocity measurements require high resolution spectra. Indeed, the Doppler shift seen at the line center is partly due to the motion of the emitting molecules. On the points of the map located east and west of the central meridian, we measure wind velocities that result from zonal and meridional winds. It should be underlined that a vertical component could also be seen in our data. However, such a component cannot be separated from the zonal and meridional component in our measurements. General circulation models predict that the zonal/meridional component is generally stronger than the vertical one (always less than $1 \mathrm{~m} \mathrm{~s}^{-1}$ ). This is the reason why we only consider the possibility that the Doppler line shifts are due to zonal/meridional winds. The other part of the frequency shift comes from the rotation of the planet. Thus, we have to take this rotation effect into account to obtain correct velocity values. A good spatial resolution is also required. This is the reason why we do not use the ${ }^{12} \mathrm{CO}(1-0)$ lines, because the beam is as large as the planet. The ${ }^{12} \mathrm{CO}(2-1)$ lines give the most precise values of the set whereas the lower signal-to-noise ratio of the ${ }^{13} \mathrm{CO}(2-1)$ observations implies greater uncertainties.

Retrieving the line-of-sight beam-integrated (LOS BI) wind velocities over the 13 point map requires a two step analysis. First, we fit the lines with a Gaussian function in order to measure the spectral shift of the core. This value, which is given with a 1- $\sigma$ fitting uncertainty, corresponds to a projection on the lineof-sight, is beam-averaged and includes the planet rotation. The rotation of Mars is modeled with a mean radius of $3389.9 \mathrm{~km}$

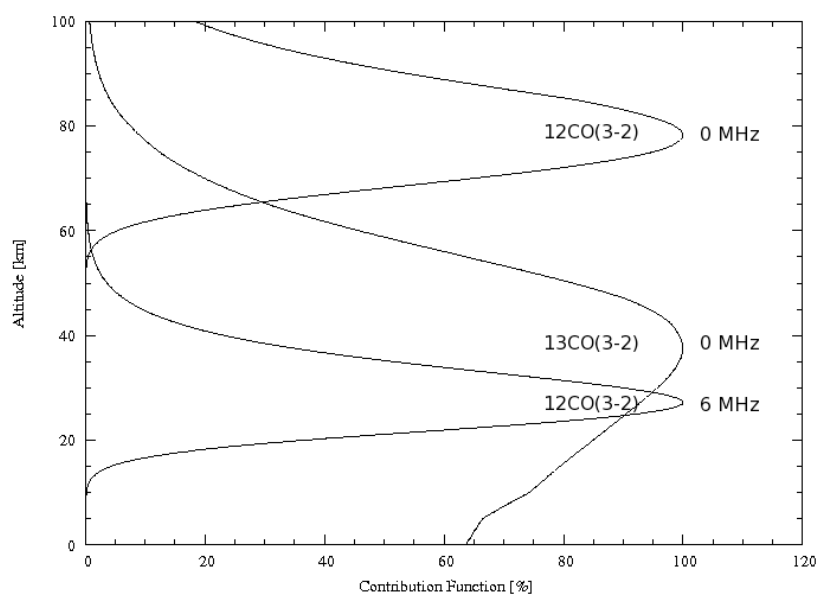

Fig. 13. Contribution function of the ${ }^{12} \mathrm{CO}(3-2)$ line at 0 and $6 \mathrm{MHz}$ from the central frequency and contribution function of the ${ }^{13} \mathrm{CO}(3-2)$ line at $0 \mathrm{MHz}$ from the central frequency in 2005 .

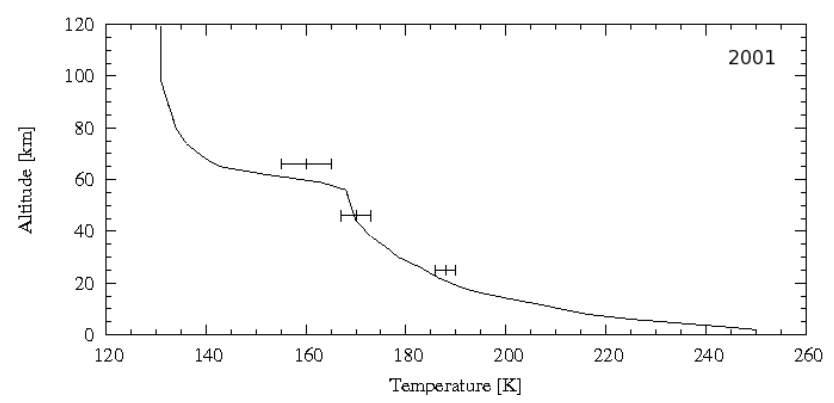

Fig. 14. Atmospheric temperature profile in 2001 derived from the observations (solid lines) and measurements from Gurwell et al. (2005) (crosses).

and a rotation time of $24.6 \mathrm{~h}$. After deriving the planet rotation component projected on the line-of-sight $\left(241 \mathrm{~m} \mathrm{~s}^{-1}\right.$ at the equatorial limbs), by taking the latitude of the SEP and the north pole angle into account, beam-averaged values of the rotation velocity are derived for each observed point and are subtracted from the observations. Thus, we obtain a map of beamaveraged zonal/meridional winds projected on the line-of-sight, as in Lellouch et al. (1991c).

There are several sources of uncertainty in the wind speed retrieval. First, we have a 1- $\sigma$ uncertainty that depends on the quality of the Gaussian fit we make to measure the spectral shift of the line center. The higher the signal-to-noise ratio, the smaller the uncertainty. This is the reason why the most precise values are derived with the ${ }^{12} \mathrm{CO}(2-1)$ lines. This uncertainty is reported in Tables 10 and 11 with the spectral shift measurements. There are other uncertainty sources that were described in Lellouch et al. (1991c) (uncertainty on the line frequency and the pressure shift of $\mathrm{CO}$ by $\mathrm{CO}_{2}$ ) and we neglect them in this model $\left(\sim 1-2 \mathrm{~m} \mathrm{~s}^{-1}\right)$. Finally, we have to check the pointing uncertainty. A robust way to validate the pointing accuracy is to draw a map of continuum values of the 13 observed points. If the continuum values are symmetrical on the eastern and western sides of the disk, then the pointing is good. The 2001 map at $230 \mathrm{GHz}$ shows a good symmetry. The difference between the limbs is about $13 \mathrm{~K}$ on the antenna temperature scale, which means that the pointing accuracy is better than 1 arcsec. On the 2005 map, the pointing offset is greater. We estimate that the mispointing is about 2 arcsec in the celestial south-east direction. We take this effect into account when computing predicted 


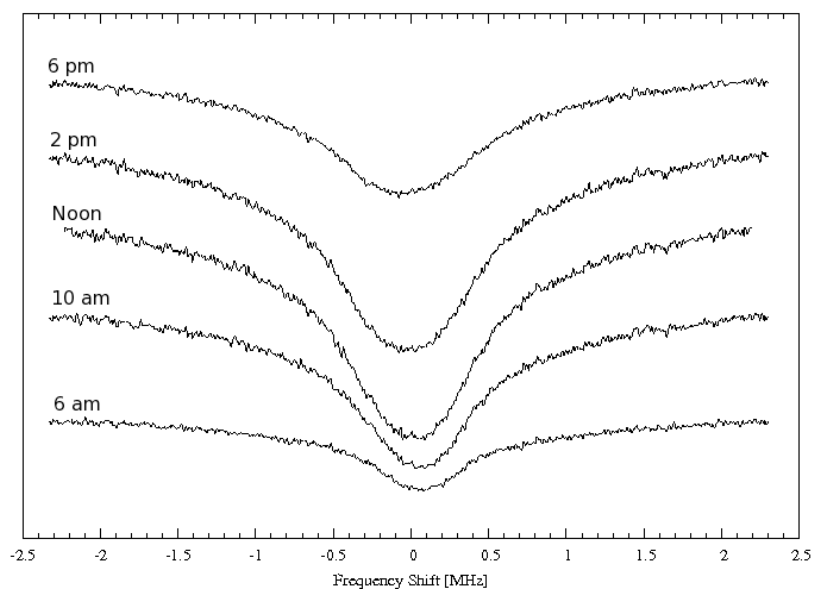

Fig. 15. ${ }^{12} \mathrm{CO}(2-1)$ line cores in 2005 for five positions on the equator. Positions are: 90W, 30W, Central Meridian (CM), 30E and 90E and correspond to $6 \mathrm{pm}, 2 \mathrm{pm}$, noon, $10 \mathrm{am}$ and $6 \mathrm{am}$ in local time.

wind velocities from the MCD database by using the values that correspond to the actually observed coordinates (coordinates assumed plus pointing shift).

\subsection{Results}

Figure 15 shows the observed ${ }^{12} \mathrm{CO}(2-1)$ high resolution spectra, in the antenna temperature scale, for the 5 positions near the equator in 2005 (90W, 30W, Central Meridian, 30E, 90E). We can clearly see the shifts due to the winds and the rotation of the planet. As previously shown, the ${ }^{12} \mathrm{CO}(2-1)$ line sounds the $70 \mathrm{~km}$ level whereas the ${ }^{13} \mathrm{CO}(2-1)$ line sounds the $40 \mathrm{~km}$ one (see Figs. 3 and 4). Observed line shifts are stored in Tables 10 and 11 for the ${ }^{12} \mathrm{CO}(2-1)$ and the ${ }^{13} \mathrm{CO}(2-1)$ lines. Positive values indicate that the winds are retrograde.

After reducing the data and plotting the retrieved LOS BI wind velocities on the planetary disk (see Figs. 16 and 17), we find retrograde winds at $70 \mathrm{~km}\left({ }^{12} \mathrm{CO}(2-1)\right)$. This easterly flow has a mean equatorial velocity of $38 \mathrm{~m} \mathrm{~s}^{-1}$ in 2001 and $77 \mathrm{~m} \mathrm{~s}^{-1}$ in 2005. These values are computed from position 4, 5, 8 and 9 measurements. We also have an easterly flow at $40 \mathrm{~km}$ in 2005, which is even stronger than at $70 \mathrm{~km}\left(95 \mathrm{~m} \mathrm{~s}^{-1}\right)$. In 2001, the winds were diverging from the central meridian at $40 \mathrm{~km}\left(\sim 55 \mathrm{~m} \mathrm{~s}^{-1}\right)$.

The velocities we obtain from the ${ }^{12} \mathrm{CO}(2-1)$ line of the easterly flows in 2001 are lower than the one obtained by Moreno et al. (2006) between 1999 and 2003. They measured easterlies with velocities of $\sim 100 \mathrm{~m} \mathrm{~s}^{-1}$. Our 2005 measurement is consistent with this measurement because of our $30-40 \mathrm{~m} \mathrm{~s}^{-1}$ error bars in 2005 (1- $\sigma$ error due to the measurement, $20 \mathrm{~m} \mathrm{~s}^{-1}$ uncertainty due to limb errors and pointing errors). Our measurements are also lower than the one obtained by Lellouch et al. (1991c) during the 1988 opposition $\left(160 \pm 80 \mathrm{~m} \mathrm{~s}^{-1}\right)$. But the solar longitude $L_{\mathrm{S}}$ was different from the $L_{\mathrm{S}}$ of our observations. Indeed, their observations were performed at $L_{S}=279^{\circ}$ (Southern summer solstice), whereas our measurements were obtained at $L_{\mathrm{S}}=180^{\circ}$ and $L_{\mathrm{S}}=316^{\circ}$. At a similar value of $L_{\mathrm{S}}=254^{\circ}$, Clancy et al. (2006) detected an easterly flow of $130-180 \mathrm{~m} \mathrm{~s}^{-1}$ from ${ }^{12} \mathrm{CO}(3-2)$ observations, consistent with Lellouch et al. (1991c). They also measured poleward meridional flows of $30-35 \mathrm{~m} \mathrm{~s}^{-1}$. Such flows are detected in our 2001 observations. The observed equatorial mean velocity is $38 \mathrm{~m} \mathrm{~s}^{-1}$, consistent with their results. On the contrary, such poleward
Table 10. Observed Doppler shifts in $\mathrm{m} \mathrm{s}^{-1}$ retrieved from the 2001 observations $\left(L_{S}=180^{\circ}\right)$. Positive values for retrograde velocities. Parenthese: $1-\sigma$ measurement uncertainties.

\begin{tabular}{ccc}
\hline \hline & ${ }^{12} \mathrm{CO}(2-1)$ & ${ }^{13} \mathrm{CO}(2-1)$ \\
Position & Doppler shift $\left[\mathrm{m} \mathrm{s}^{-1}\right]$ & Doppler shift $\left[\mathrm{m} \mathrm{s}^{-1}\right]$ \\
\hline 1 & $23(2)$ & $41(6)$ \\
2 & $30(2)$ & $60(9)$ \\
3 & $47(3)$ & $70(16)$ \\
4 & $75(2)$ & $106(6)$ \\
5 & $95(2)$ & $162(7)$ \\
6 & $18(3)$ & $32(8)$ \\
7 & $10(4)$ & $85(16)$ \\
8 & $-57(3)$ & $-71(7)$ \\
9 & $-112(3)$ & $-126(8)$ \\
10 & $-76(3)$ & $-49(12)$ \\
11 & $90(3)$ & $117(9)$ \\
12 & $74(3)$ & $124(8)$ \\
13 & $-70(3)$ & $-51(15)$ \\
\hline
\end{tabular}

Table 11. Same caption as Table 10 for the 2005 observations $\left(L_{\mathrm{S}}=\right.$ $316^{\circ}$.

\begin{tabular}{ccc}
\hline \hline & & \\
Position & Doppler shift $\left[\mathrm{m} \mathrm{s}^{-1}\right]$ & $\begin{array}{c}{ }^{13} \mathrm{CO}(2-1) \\
\text { Doppler shift }\left[\mathrm{m} \mathrm{s}^{-1}\right]\end{array}$ \\
\hline 1 & $-26(3)$ & $0(7)$ \\
2 & $-40(2)$ & $3(8)$ \\
3 & $-42(2)$ & $-14(19)$ \\
4 & $14(3)$ & $35(6)$ \\
5 & $37(4)$ & $49(7)$ \\
6 & $-9(3)$ & $8(7)$ \\
7 & $1(4)$ & $15(11)$ \\
8 & $-66(2)$ & $-32(8)$ \\
9 & $-91(2)$ & $-12(15)$ \\
10 & $-91(2)$ & $-6(20)$ \\
11 & $14(3)$ & $22(9)$ \\
12 & $30(4)$ & $48(8)$ \\
13 & $-53(3)$ & $4(12)$ \\
\hline
\end{tabular}

flows are not observed on the 2005 data, but flows converging towards the SEP are.

\subsection{Using the Mars Climate Database to compute predicted wind velocities - comparison with observational data}

We use the same square grid and the same corresponding vertical profiles as the one used to compute thermal profiles, because the local zonal and meridional wind velocities are also computed from the atmemcd subroutine. On each line-of-sight, we obtain the zonal and meridional wind components for each altitude. To derive the measured component on this line-of-sight, we need to average these velocity vertical profiles by using the contribution function of the spectral line we are analysing. Once we have a zonal and meridional velocity value for each line-of-sight of our grid, we have to project them on the corresponding line-of-sight. Then, we add these contributions and convolve the results by the antenna pattern, pointed at the location of the observation we are interested in, in order to obtain LOS BI predictions. To obtain the 12 other values of the map, we center the antenna pattern on each point.

For the winds, we have the same problem as the one we have with the temperature vertical profiles above the planetocentric points of our map. Once again, when interested in points close to the limb, the planetocentric information available from the MCD is not exactly the information on the line-of-sight, because 
(a)

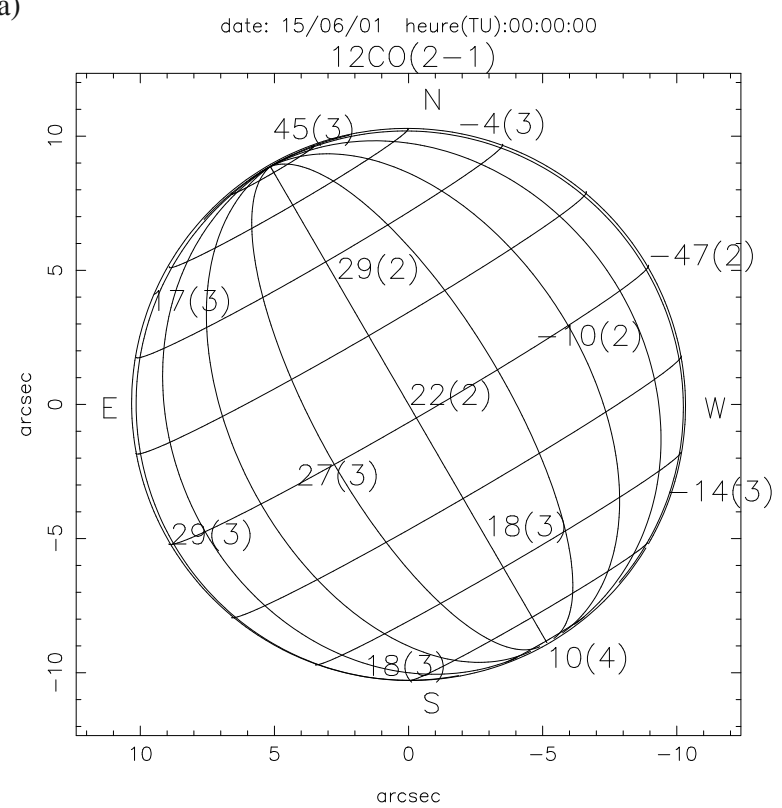

(b)

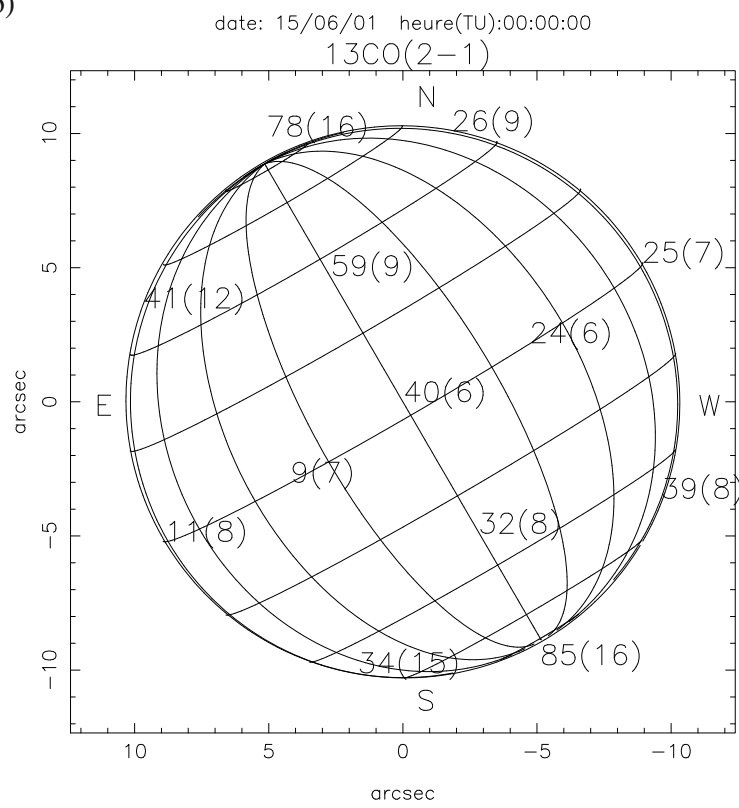

Fig. 16. LOS BI wind velocities $\left[\mathrm{m} \mathrm{s}^{-1}\right]$ for the a) ${ }^{12} \mathrm{CO}(2-1)$ and b) ${ }^{13} \mathrm{CO}(2-1)$ observations in 2001. Celestial east/west directions. Parenthese: $1-\sigma$ fitting uncertainties. Additional uncertainty on each point: $20 \mathrm{~m} \mathrm{~s}^{-1}$ for ${ }^{12} \mathrm{CO}(2-1)$ measurements and $30-40 \mathrm{~m} \mathrm{~s}^{-1}$ for ${ }^{13} \mathrm{CO}(2-1)$ measurements.

of the viewing angle. Yet, we also choose to use the values given for the planetocentric points and use them on the lines-of-sight, because the uncertainty is small $\left(\leq 20 \mathrm{~m} \mathrm{~s}^{-1}\right)$ on the points that are on the edge of the planetary disk.

The MCD MY24 and Warm scenarios predict velocities that are all in the same range, the differences on a given point of the map rarely exceeding $20 \mathrm{~m} \mathrm{~s}^{-1}$. This is the reason why we only present results from the MY24 scenario. Figures 18 and 19 show the LOS BI wind velocity predictions of the MY24 scenario of the MCD. On each color map, the values of the wind velocities are displayed at each observed position.

The MCD MY24 scenario gives predictions that are in good agreement with the $2001{ }^{12} \mathrm{CO}(2-1)$ observations (see Figs. 16a and $18 \mathrm{a}$ ), all predicted numbers being within the error bars of the (a)

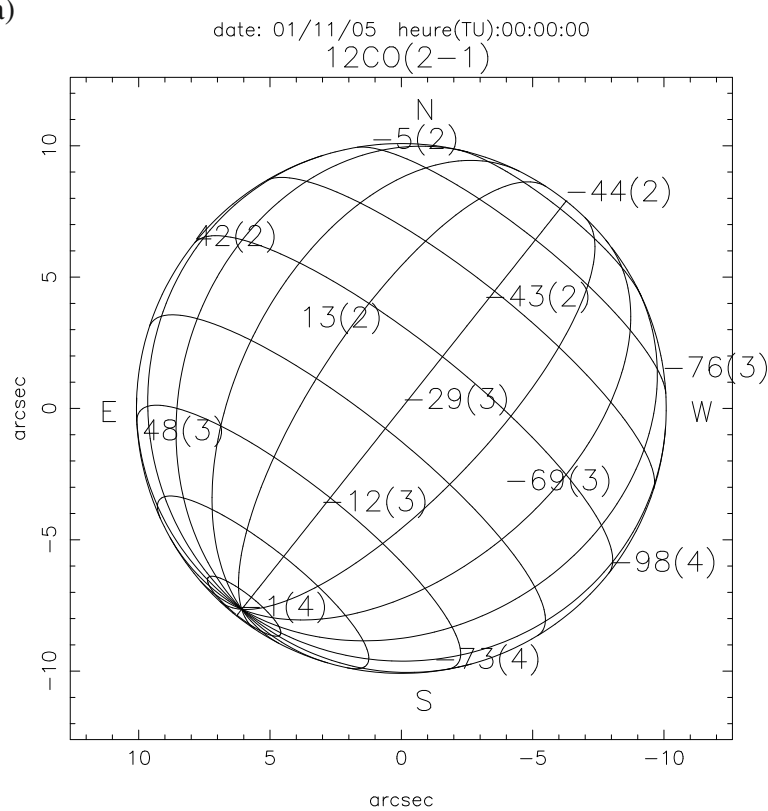

(b)

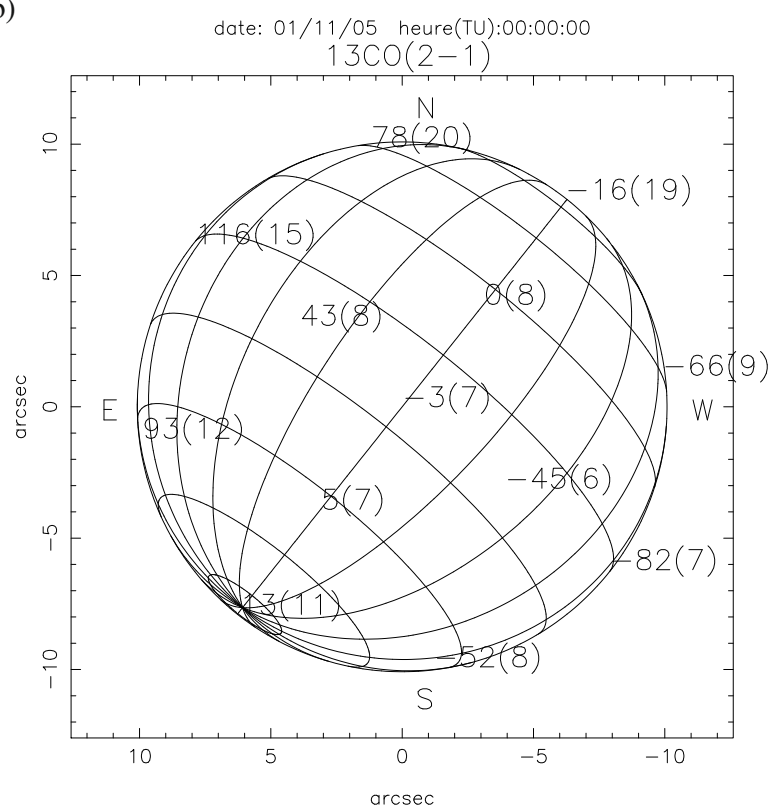

Fig. 17. LOS BI wind velocities $\left[\mathrm{m} \mathrm{s}^{-1}\right]$ for the a) ${ }^{12} \mathrm{CO}(2-1)$ and b) ${ }^{13} \mathrm{CO}(2-1)$ observations in 2005 . Celestial east/west directions. Parenthese: 1- $\sigma$ fitting uncertainties. Additional uncertainty on each point: $20 \mathrm{~m} \mathrm{~s}^{-1}$ for ${ }^{12} \mathrm{CO}(2-1)$ measurements and $30-40 \mathrm{~m} \mathrm{~s}^{-1}$ for ${ }^{13} \mathrm{CO}(2-1)$ measurements.

observations (1- $\sigma$ uncertainty due to the measurement and an additional uncertainty up to $20 \mathrm{~m} \mathrm{~s}^{-1}$ ). Some mismatching occurs in the northern hemisphere along the central meridian, where $10 \mathrm{~m} \mathrm{~s}^{-1}$ southward winds are predicted. Also, the ${ }^{13} \mathrm{CO}(2-1)$ observations (Fig. 16b) are consistent with the MCD MY24 scenario predictions (see Fig. 18b), except along the central meridian. We observe poleward flows that are not predicted. Part of this discrepency may come from the larger error bars on the measurements $\left(\sim 30-40 \mathrm{~m} \mathrm{~s}^{-1}\right)$.

The MCD MY24 scenario predictions are generally consistent with the ${ }^{12} \mathrm{CO}(2-1)$ measurements from 2005 (see Figs. 17a and 19a), the only differences occuring on the celestial western part of the disk where the predicted zonal winds are too strong by a factor of $2-3$. The celestial eastern part of the disk is well 


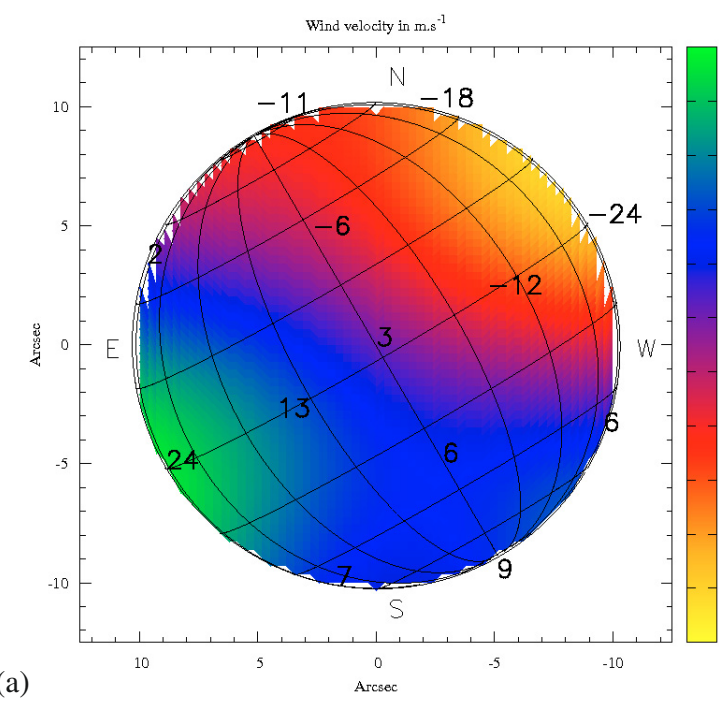

(a)

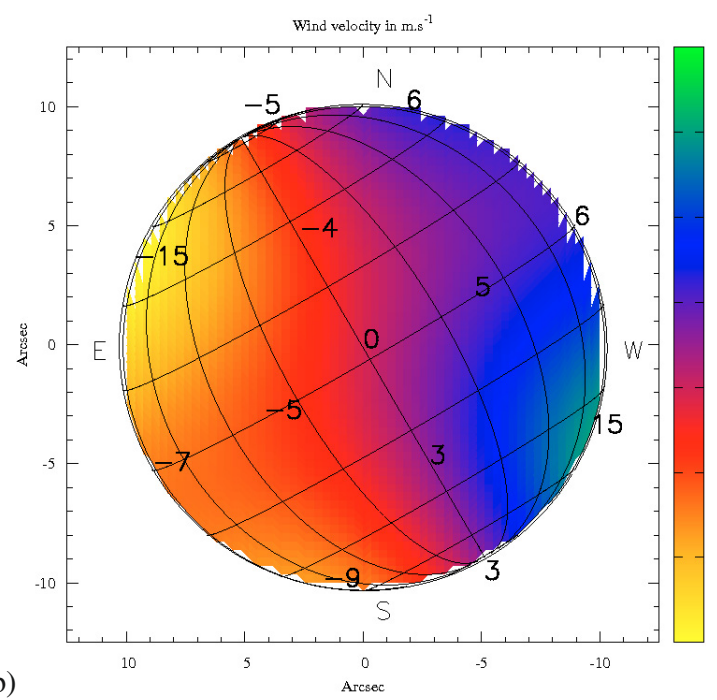

Fig. 18. Wind velocities predictions from the MCD MY24 scenario $\left[\mathrm{m} \mathrm{s}^{-1}\right.$ ] for a) the ${ }^{12} \mathrm{CO}(2-1)$ and b) the ${ }^{13} \mathrm{CO}(2-1)$ in 2001 . The numbers correspond to the velocities on the 13 points of the observed map. Celestial east/west directions.

predicted within an uncertainty of $20 \mathrm{~m} \mathrm{~s}^{-1}$. The meridional component, which is seen on the central meridian, does not match the predictions in the northern hemisphere, where we observe a southward flow. The results are very similar when taking a 2 arcsec mispointing towards the south-east celestial direction into account, for the geometry. The wind velocity predictions for the altitudes sounded by the ${ }^{13} \mathrm{CO}(2-1)$ line (Fig. 19b) are stronger than the observed (Fig. 17b) ones by a factor of 2-4 on the western side of the disk and they are even stronger on the eastern side of the disk.

Generally, the MCD gives good predictions for wind velocities at the time of our observations and at the spatial scale given by the beam of the antenna we have used. Some discrepencies appear at $40 \mathrm{~km}$, but our low signal-to-noise ratio in the ${ }^{13} \mathrm{CO}(2-1)$ observations may, at least partly, explain these discrepancies. A higher spatial resolution is needed to confirm that the MCD gives satisfactory wind predictions at a more precise scale on the planet. Interferometric observations should help this (Moreno et al. 1999, 2001, 2006).

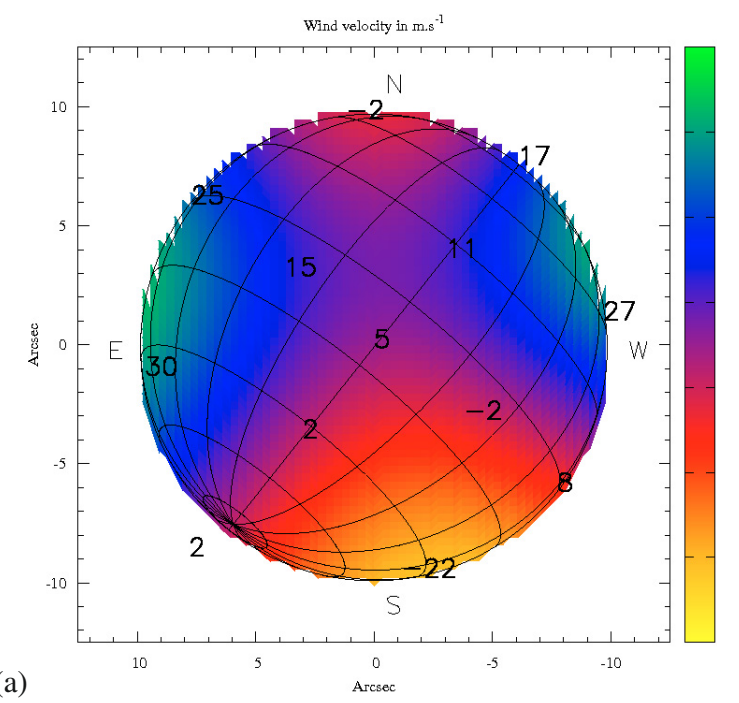

(a)

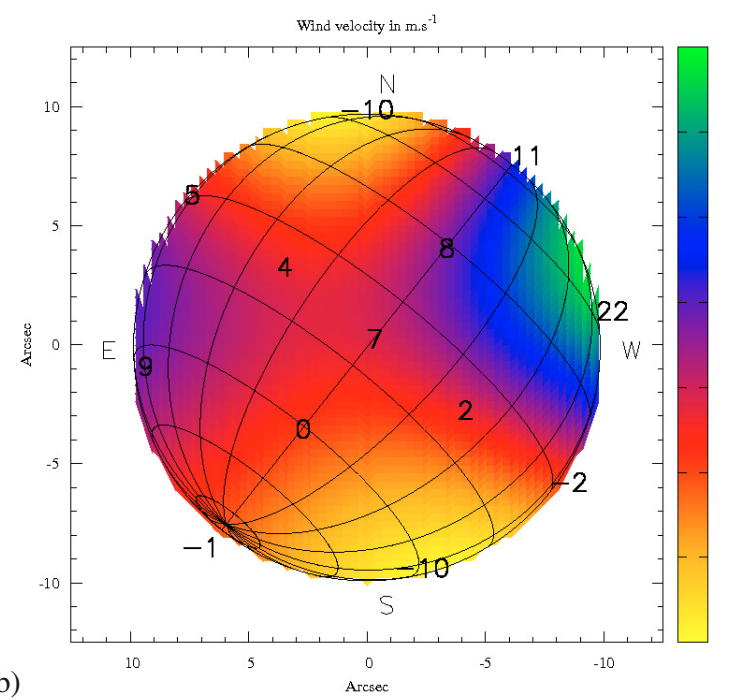

Fig. 19. Same caption as Fig. 18 for 2005. Celestial east/west directions. Taking a pointing error of 2 arcsec in the south-east direction into account would result in reducing the velocities of the western limb by $\sim 20 \mathrm{~m} \mathrm{~s}^{-1}$ and increasing the eastern limb velocities by $\sim 20 \mathrm{~m} \mathrm{~s}^{-1}$.

\section{Conclusion}

This work on low/high resolution ${ }^{12} \mathrm{CO}$ and ${ }^{13} \mathrm{CO}$ millimeter wavelength spectra shows that it is possible to retrieve surface and atmospheric parameters with good precision. Our results are in general agreement with observations carried out from other facilities (ground and space-based). We showed that the MCD model, the Gurwell et al. (2005) or the Clancy et al. (2006) observations give temperature profiles above $30 \mathrm{~km}$ that are different from ours. When comparing MCD predictions to the data, the Warm scenario gives the best fits at low spectral resolution to the observations, but discrepencies are seen in the high spectral resolution data. A sudden decrease of temperature is seen at $55 \mathrm{~km}$ in 2001 and at $45 \mathrm{~km}$ in 2005, that is not predicted by any of the tested scenarios of the MCD (MY24, Dust storm and Warm scenarios). The MCD MY24 model features a thermal inversion layer between 40 and $80 \mathrm{~km}$, which is not observed in the data we present here. This strong decrease of the temperature which is observed above $45-55 \mathrm{~km}$ can only be seen with the high resolution spectra. This underlines the importance of high spectral resolution data to retrieve thermal profiles correctly. The precise retrieval of thermal profiles will be of key importance in 
order to constrain the vertical profiles of the species that will be observed or newly detected with the Herschel Space Observatory and interferometers.

Also, such data give us the opportunity to measure wind velocities from line center Doppler shifts. Wind velocities were retrieved from ${ }^{13} \mathrm{CO}(2-1)$ observations, giving access to the $40 \mathrm{~km}$ layer of the atmosphere. The MCD MY24 and Warm scenario predictions are in agreement with the observations of ${ }^{12} \mathrm{CO}(2-1)$ in 2001 and 2005. Some discrepencies appear at $40 \mathrm{~km}$, when comparing our ${ }^{13} \mathrm{CO}(2-1)$ to the $\mathrm{MCD}$ predictions. The zonal wind velocities, obtained from ${ }^{12} \mathrm{CO}$ observations, are generally not as strong as the one observed by Moreno et al. (2006) and Clancy et al. (2006) in 2003. Easterly flows are seen at $70 \mathrm{~km}$ with a mean equatorial velocity of $38 \mathrm{~m} \mathrm{~s}^{-1}$ in 2001 and $77 \mathrm{~m} \mathrm{~s}^{-1}$ in 2005 .

\section{References}

Billebaud, F., Maillard, J. P., Lellouch, E., \& Encrenaz, T. 1992, A\&A, 261, 647

Billebaud, F., Rosenqvist, J., Lellouch, E., et al. 1998, A\&A, 333, 1092

Chassefiere, E., Blamont, J. E., Krasnopol'Skii, V. A., et al. 1992, Icarus, 97, 46

Clancy, R. T., Muhleman, D. O., \& Jakosky, B. M. 1983, Icarus, 55, 282

Clancy, R. T., Sandor, B. J., Moriarty-Schieven, G. H., \& Smith, M. D. 2006, in Mars Atmosphere Modelling and Observations, ed. F. Forget, M. A. Lopez-Valverde, M. C. Desjean, et al., 135

Clancy, R. T., Sandor, B. J., Wolff, M. J., et al. 2000, J. Geophys. Res., 105, 9553

Encrenaz, T., Fouchet, T., Melchiorri, R., et al. 2006, A\&A, 459, 265

Encrenaz, T., Lellouch, E., Paubert, G., \& Gulkis, S. 2001, Planet. Space Sci., 49, 731

Forget, F., Hourdin, F., Fournier, R., et al. 1999, J. Geophys. Res., 104, 24155
Forget, F., Millour, E., Lebonnois, S., et al. 2006, in Mars Atmosphere Modelling and Observations, ed. F. Forget, M. A. Lopez-Valverde, M. C. Desjean, et al., 128

Forget, F., Lebonnois, S., Angelats I Coll, M., et al. 2007, J. Geophys. Res., submitted

Gurwell, M. A., Bergin, E. A., Melnick, G. J., et al. 2000, ApJ, 539, L143

Gurwell, M. A., Bergin, E. A., Melnick, G. J., \& Tolls, V. 2005, Icarus, 175, 23

Kaplan, L. D., Connes, J., \& Connes, P. 1969, ApJ, 157, L187

Krasnopolsky, V. A. 1993, Icarus, 101, 33

Krasnopolsky, V. A. 2003, J. Geophys. Res. (Planets), 108, 5010

Lellouch, E., Gerin, M., Combes, F., Atreya, S., \& Encrenaz, T. 1989, Icarus, 77, 414

Lellouch, E., Encrenaz, T., Phillips, T., Falgarone, E., \& Billebaud, F. 1991a, Planet. Space Sci., 39, 209

Lellouch, E., Paubert, G., \& Encrenaz, T. 1991b, Planet. Space Sci., 39, 219

Lellouch, E., Rosenqvist, J., Goldstein, J. J., Bougher, S. W., \& Paubert, G. 1991c, ApJ, 383, 401

Lewis, S. R., Collins, M., Read, P. L., et al. 1999, J. Geophys. Res., 104, 24177

Mellon, M. T., Jakosky, B. M., Kieffer, H. H., \& Christensen, P. R. 2000, Icarus, 148,437

Moreno, R., Guilloteau, S., Lellouch, E., et al. 1999, in BAAS, 31, 1149

Moreno, R., Guilloteau, S., Lellouch, E., et al. 2001, in BAAS, 33, 1072

Moreno, R., Lellouch, E., Encrenaz, T., et al. 2006, in Mars Atmosphere Modelling and Observations, ed. F. Forget, M. A. Lopez-Valverde, M. C. Desjean, et al., 134

Nair, H., Allen, M., Anbar, A. D., Yung, Y. L., \& Clancy, R. T. 1994, Icarus, 111,124

Owen, T., Biemann, K., Biller, J. E., et al. 1977, J. Geophys. Res., 82, 4635

Pickett, H. M., Poynter, I. R. L., Cohen, E. A., et al. 1998, J. Quant. Spectrosc. Radiat. Transf., 60, 883

Shah, K. P., Muhleman, D. O., \& Berge, G. L. 1991, Icarus, 93, 96

Smith, M. D. 2004, Icarus, 167, 148

Sonnabend, G., Sornig, M., Krötz, P. J., Schieder, R. T., \& Fast, K. E. 2006, Geophys. Res. Lett., 33, 18201

Sprague, A. L., Boynton, W. V., Kerry, K. E., et al. 2004, Science, 306, 1364

Theodore, B., Lellouch, E., Chassefiere, E., \& Hauchecorne, A. 1993, Icarus, 105,512

Varanasi, P. 1975, J. Quant. Spectrosc. Radiat. Transf., 15, 191 